\title{
A genome-wide CRISPR/Cas phenotypic screen for modulators of DUX4 cytotoxicity reveals screen complications
}

Ator Ashoti ${ }^{*}$, Francesco Limone ${ }^{2,5^{*}}$, Melissa van Kranenburg ${ }^{1}$, Anna Alemany $^{1}$, Mirna Baak ${ }^{1}$, Judith Vivié ${ }^{1,3}$, Federica Piccioni ${ }^{8}$, Menno

Creyghton ${ }^{1,6}$, Kevin Eggann $2,5^{* *}$, Niels Geijsen ${ }^{1,4^{* *}}$

${ }^{1}$ Hubrecht Institute, Developmental Biology and Stem Cell Research, Utrecht, Netherlands

2Department of Stem Cell and Regenerative Biology, Harvard University Cambridge, MA, USA

${ }^{5}$ Stanley Center for Psychiatric Research, Broad Institute of MIT and Harvard, Cambridge, MA, USA

${ }^{3}$ Single Cell Discoveries, Utrecht, Netherlands

${ }^{4}$ Leiden University Medical Center, Leiden, The Netherlands

${ }^{6}$ Erasmus University Medical Centre, Rotterdam, The Netherlands ${ }^{8}$ Broad institute, Cambridge, MA, USA

* Equal contribution

${ }^{* \star}$ Correspondence: $\underline{\text {.geijsen@lumc.nl, eggan@mcb.harvard.edu }}$

\section{Acknowledgements}

This study was supported by Stichting FSHD and the SingelSwim Utrecht. The authors like to thank Nune Schelling, Peng Shang, Stefan van der Elst, en Reinier van der Linden for excellent technical assistance. 


\section{Abstract}

29 Facioscapulohumeral muscular dystrophy (FHSD), a fundamentally complex muscle

30 disorder that thus far remains untreatable. As the name implies, FSHD starts in the muscles of the face and shoulder gridle. The main perturbator of the disease is the pioneer transcription factor DUX4, which is misexpressed in affected tissues due to a failure in epigenetic repressive mechanisms. In pursuit of unraveling the underlying mechanism of FSHD and finding potential therapeutic targets or treatment options, we performed an exhaustive genome-wide CRISPR/Cas9 phenotypic rescue screen to identify modulators of DUX4 cytotoxicity. We found no key effectors other than DUX4 itself, suggesting treatment efforts in FSHD should be directed towards its direct modulation.

The screen did however reveal some rare and unexpected Cas9-induced genomic events, that may provide important considerations for planning future CRISPR/Cas9 knock-out screens.

\section{Introduction}

Facioscapulohumeral muscular dystrophy (FSHD) is an autosomal dominant degenerative muscle disease. It's one of the most prevalent neuromuscular disorder ${ }^{1}$, characterized by progressive and asymmetric muscle weakness which generally starts in facial muscles, and then slowly progresses to muscles of the shoulders, upper limbs and eventually the lower extremities ${ }^{2}$. Age of onset is highly variable, but calculations based on a 122 case study demonstrates that the mean age of onset is in the early twenties $(21-23)^{3}$. The primary cause of the disease is the misexpression of the double homeobox 4 (DUX4) transcription factor, due to failure in epigenetic silencing ${ }^{3-6}$. DUX4 is normally expressed early in development in the cleavage stage embryo ${ }^{7,8}$, in the adult testis ${ }^{6}$ and in the thymus ${ }^{9}$. De-repression of DUX4 in muscle activates a large cascade of events, triggering the activation of many pathways $\mathrm{s}^{8,10-19}$, with target genes being involved in biological processes such as RNA splicing and processing (DBR110,20-22, CWC1510,20,22, PNN10,21, CLP110,21,22, TFIP1110,20-22), spermatogenesis 
59 (CCNA1 ${ }^{10,20-22}, \quad$ ZNF296 ${ }^{10,20-22}$, TESK2 $\left.{ }^{10,20,21}\right)$, early embryonic development (ZSCAN4 ${ }^{10,20-22}$, LEUTX ${ }^{20-22}$, STIL $\left.{ }^{10,20,21}\right)$, protein processing and degradation (SIAH110,20-22, RHOBTB1 10,20,21, TRIM3610,20,21), and cell motility and migration (CXCR410,20,21, ROCK110,21, SNAI1 10,20-22).

We hypothesized that of one or more factors downstream of DUX4 expression are responsible for the rapid apoptotic response that follows DUX4 induction. Knowing if there are key downstream targets of DUX4 can have important clinical applications as they could direct intelligent therapeutic design. We tested this hypothesis by performing a genome wide CRISPR/Cas9 knockout screen. CRISPR/Cas9 which is now a highly popular and widely used genome editing technique, was initially discovered as the adaptive immune system of bacteria, to protect against viral infection ${ }^{23,24}$. Although not the first genome editing method, CRISPR/Cas9 has proven to be much more user friendly due to its easy manipulability, and being more cost-, labor- and time-efficient compared to its predecessors: transcription activator-like effector nucleases (TALENs) ${ }^{25-28}$ and ZINC-fingers nucleases (ZFNs) ${ }^{29-33}$. Its ability to knock-out any gene by creating a double stranded break ${ }^{34-36}$ in such an easy manner, makes this technique very suitable for genomewide loss off function studies. The advantages and ease-of-use of the CRISPR/Cas9 technology inspired us to perform a genome wide screen on a FSHD in-vitro model, to find potential modulators that contribute or aggravate the FSHD pathophysiology. Successful performance of a FSHD genome-wide screen will critically depend on the cell system being used. The cells should be highly proliferative, easily transfected and display a robust DUX4-induced phenotype. Fortunately, DUX4 is a so-called pioneer factor $^{49,50}$, capable of regulating its target genes independent of their chromatin-state. The network of genes activated by pioneer factors is therefore less affected by cellular identity. Indeed, Jones and colleagues have demonstrated that DUX4 activates the same downstream target genes in B-lymphocytes as previously identified in skeletal muscle myoblasts ${ }^{51,52}$. Using an adherent leukemic cell line that is frequently used for genome-wide screening purposes (HAP153,54), we performed an exhaustive CRISPR knockout screen to identify factors that could mitigate DUX4-induced cytotoxicity. We inserted a doxycycline-inducible DUX4 transgene into the HAP1 cells ${ }^{53,54}$ to generate 
screened for modulators of DUX4 cytotoxicity. Our results suggest that no single gene knockout is capable of rescuing DUX4-triggered apoptosis in our transgene model system.

This study does however, provide some interesting insight into critical parameters that need to be considered when executing a genome-wide CRISPR screen.

97

98

\section{Results}

\section{Generation and validation of a DUX4 inducible cell line}

In order to perform a successful genome-wide screen, a cellular model is required that is highly proliferative, easily transfected and which displays a robust phenotype. HAP1 cells display all these characteristics and have been used extensively in a wide variety of functional screens ${ }^{54,56-64}$. The cells were initially reported to be near-haploid ${ }^{53,54}$, but subsequently rediploidized65,66. These rediploidized cells were used to generate the inducible DUX4 cell line. Since even low levels of DUX4 expression can efficiently induce apoptosis $6,20,67$, we needed to circumvent premature DUX4 toxicity caused by leaky expression of the Tet-On system ${ }^{68,69}$ during the generation of the cell line. To avoid this, we inserted a LoxP-DsRed-LoxP stop-cassette in between the Tet-operator and a DUX4 transgene. The DUX4 transgene element itself consisted of the three exons and the two introns of DUX4 including the polyA terminal sequence (haplotype $\left.4 \mathrm{~A} 161^{70}\right)$. After stable integration of the construct, the stop-cassette was removed using CRE recombinase, thereby placing DUX4 under the control of the Tet operator (Fig. 1A). 80 monoclonal lines were derived by single cell flow-cytometry sorting, of which 1 demonstrated DUX4 induction and robust cell death upon doxycycline addition (Fig. 1B), which we named 'DUX4-inducible expression' (DIE) cells.

To identify the sites of integration of the rtTA/BlastR and DUX4/PuroR transgenes in the DIE cells, we performed targeted locus amplification (TLA) ${ }^{71}$. TLA analysis showed a single integration site for both the rtTA and DUX4 transgenes (Fig. 1C). The DUX4 cassette integrated in the p-arm of chromosome 19, within the MAST1 gene, and the 
122 rtTA cassette integrated in the MGAT4B gene which is located at the end of

123 chromosome 5q.

124 To further analyze the functional effect of DUX4 induction, DIE cells were stained with 125 AnnexinV-Alexa Fluor 488 and propidium iodine (PI) (Fig. S2). As shown in the 126 supplementary video's, DIE cells stained positive for the apoptotic marker AnnexinV, 127 during 12 hours of doxycycline exposure. To show that the apoptotic phenotype was 128 dependent on the induction of the DUX4 transgene, we targeted the DUX4 transgene using CRISPR/Cas9 and 2 independent guide RNAs (gRNAs), one targeting the C-

130 terminal domain of DUX4 and the other close to the polyA tail of DUX4. RT-qPCR and 131 Western blot (WB) analysis of the DIE and the DIE-DUX4 knockout (DIE-KO) cells 132 demonstrated successful knockout of the DUX4 transgene at RNA and protein level 133 (Fig. 1D). In addition, CRISPR/Cas9 targeting of the DUX4 transgene successfully 134 rescued the DIE cells from apoptosis upon doxycycline administration, demonstrating 135 that apoptosis upon doxycycline induction in the DIE line is mediated by DUX4 (Fig. 136 1E). DUX4 induction in the DIE cells also resulted in induction of its known 137 downstream target genes (LEUTX, ZSCAN4, PRAMFE1 and ZNF217) (Fig. 1F), 138 demonstrating that inducing DUX4 expression induces downstream target genes that are also induced by endogenous DUX4.

141 Figure 1. Creation and validation of the DIE cell line. (A) Schematic representation of the i) rtTA 142 construct, ii) the inducible LSL-DUX4 cassettes, and the inducible DUX4 cassette upon removal of the 143 stop cassette (LSL) through CRE recombinase. (B) Phase contrast images of DIE cells i) without 144 doxycycline exposure and ii) with $24 \mathrm{~h}$ of doxycycline exposure. (C) Schematic representation of 145 transgene integration sites within human genome, by TLA analysis. The inducible DUX4 cassette maps 146 back to the $p$-arm of chromosome 19, and the rtTA transgene maps back to the end of the q-arm of 147 chromosome 5. (D) Expression of DUX4 mRNA and protein in HAP1, DIE and DIE-KO cells with or 148 without doxycycline admission, as detected by qRT-PCR and western blot analysis. $\beta$-actin served as 149 a loading control in the western blot. (E) Phase contrast images of doxycycline exposed DIE cells which 150 were transduced with either i) only Cas9 protein, or ii) Cas9 protein and DUX4 sgRNA. Dead cells were 151 removed by a DPBS wash to expose the surviving population. (F) mRNA expression of known 152 downstream targets of DUX4 in HAP1, DIE and DIE-KO cells with or without doxycycline admission, as measured by qRT-PCR. 

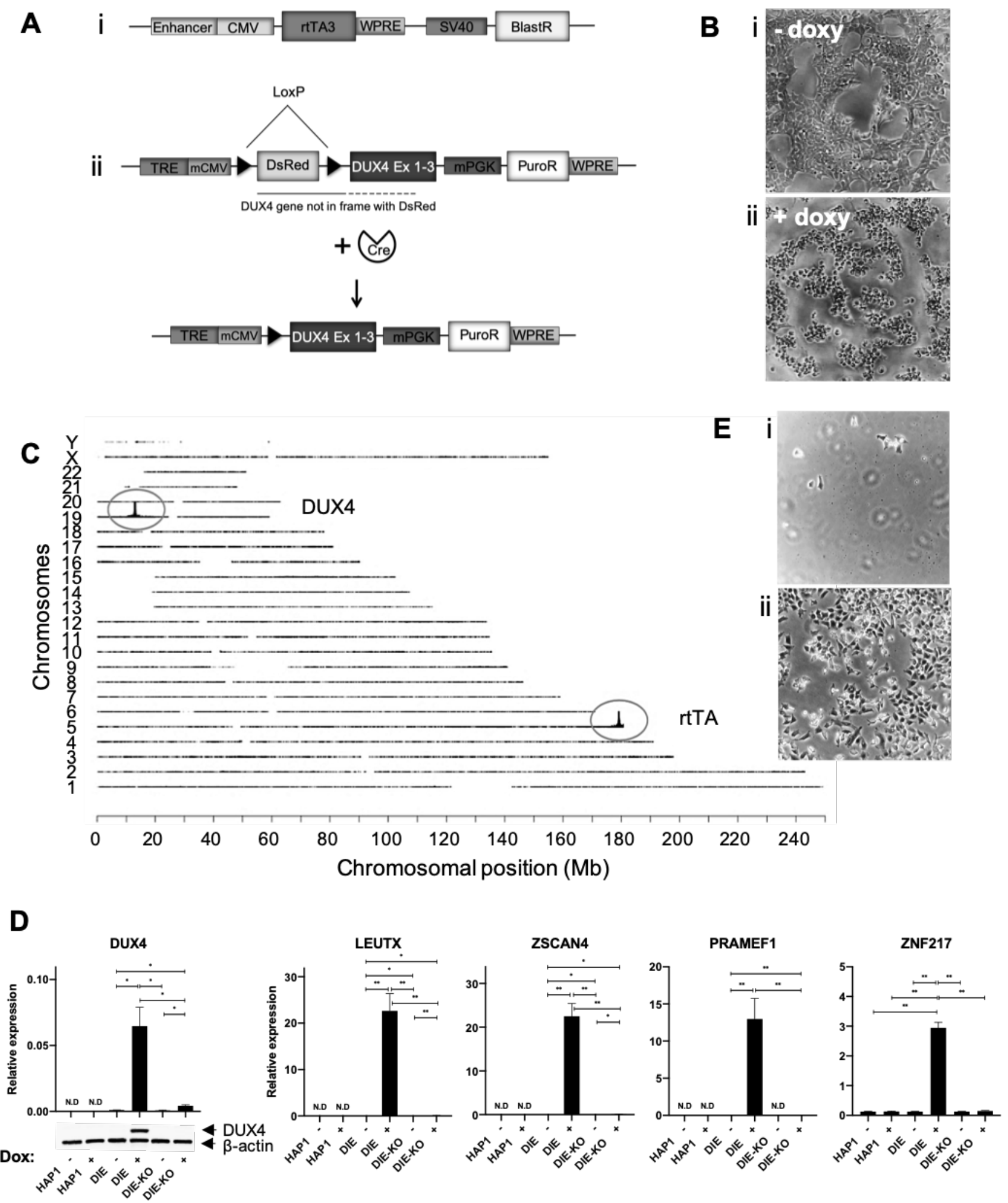

\section{DUX4 gene expression signature in DIE cells}

156 Next we analyzed the downstream transcriptional changes that were induced by DUX4

157 in the DIE cells with RNA sequencing. We compared 4 induced and uninduced

158 technical replicates of two lines; the DIE line, and the DIE-KO line. As shown in Fig. 
2A, DUX4 induction resulted in progressive temporal changes in gene expression. To visualize the magnitude and speed of the DUX4-induced transcriptional changes, we plotted the log2fold change of all detected transcripts against the density of differentially expressed genes at any given log2fold change (Fig. 2B). As shown, DUX4 induction results in a profound and progressive increase in the number of differentially expressed genes; with 64 differentially expressed transcripts at 4.5 hours post DUX4 induction and 467 differentially expressed transcripts at 8.5 hours (Fig. 2B and $2 \mathrm{C}$ ). The number of genes of which the expression is induced is greater than those with reduced expression levels, consistent with DUX4's role as an activating pioneering factor ${ }^{49}$ (Fig. $2 \mathrm{~B}$ ). This is further reflected by the differential expression analysis, demonstrating an increase in upregulated genes in both induced DIE samples compared to uninduced DIE sample [Padj value $\leq 0.01$, Log2foldchange $\geq 1$ ] (Fig. 2C and D, Supplementary Table S1). Most differentially expressed genes are shared between the two induced samples (Fig. 2C). Among the upregulated genes are well known downstream targets of DUX4, including LEUTX, ZSCAN4, PRAMEF1 and ZNF217 (Fig. 2E). We next used Enrichr72,73 to search for studies that have detected similar transcriptome changes as those found in our DUX4-induction experiment, and found one other DUX4 study that has been entered into the Enrichr database (GSE33799) ${ }^{10}$ that shows high similarities to our data. Based on Enrichr entries, the upregulated genes in induced DIE cells are linked to DUX4 activity [Log10 $(P$-value $)=100.3$, as are the downregulated genes [-Log10 $(P$-value $)=3.8$ ] (Fig. 2F and table S2). Interestingly, PAX5 appears to also be linked to the upregulation of some of the same genes as DUX4 (21 and 24 overlapping genes). The Paired box (PAX) family of genes has previously been linked to FSHD and DUX4, and could explain this overlap in gene activation $17,74,75$. Additionally, genes are often regulated by more than 1 transcription factor, which could also explain the shared target genes. We next compared the list of differentially expressed genes (DIE_8.5h) with 4 other studies that have previously explored the DUX4 transcriptional network (Geng, Rickard, Jagannathan and Heuvel)10,20-22. As shown in Figure 2G, we observed a high percentage of overlap between our dataset and those previously reported. The overlap between the upregulated genes is greater than the overlap that can be seen in the downregulated genes. This applies not only for the overlaps seen 
191 between our data and the other datasets, but also between the 4 other datasets (Fig.

192 2G and table S3). This confirms the believe that DUX4 is an activating factor, and that

193 the down regulated genes might be more influenced by cell type.

194 Overlapping data seen here are likely an underrepresentation, due to the presence of 195 gene families containing paralogs and pseudogenes in either reference genome 196 databases, which can lead to multi-mapped or ambiguous reads ${ }^{76}$. To conclude, data 197 shown here strongly suggest that in our DIE cell system, DUX4 induces transcriptional changes similar to those found in FSHD muscle.

200 Figure 2. RNA-sequencing data reveals differentially expressed genes upon DUX4 expression.

201 (A) Heatmap showing differentially expressed (DE) genes between samples, with gene clusters (color coded) on y-axis, and samples on the X-axis. (B) Gene density plot demonstrating the effects of DUX4 activation on the transcriptome of the DIE cell line. DUX4 activation results in an increase of DE genes compared to uninduced DIE cells, as indicated by the bell shape widening and shortening. (C) Venn diagram showing the overlap and the number of DE expressed genes at $4.5 \mathrm{~h}$ and $8.5 \mathrm{~h}$ of doxycycline induction (Adjusted P-value $\leq 0.01$, and absolute Log2FC $\geq 1$ ). (D) Scatter plots of gene expression (RPM: reads per million) of induced DIE cells versus uninduced DIE cells. Left two panels demonstrate uninduced DIE cells (DIE_Oh) on the $\mathrm{x}$-axis versus uninduced or induced DIE-KO samples (KOOh and $\mathrm{KO} .5 \mathrm{~h}$ ) on the $\mathrm{y}$-axis. Right two panels compare the uninduced DIE cells on the $\mathrm{x}$-axis with induced

210 DIE samples (4.5h and 8.5h) on the y-axis. Green and red points represent the DE genes with an

211 Adjusted $P$-value $\leq 0.01$, and absolute $\log 2 F C \geq 1$. Green points represent upregulated genes, and the

212 red points represent downregulated genes. (E) Count plots showing UMI and between sample

213 normalized transcript counts of 4 known DUX4 targets genes: LEUTX, ZSCAN4, PRAMEF1 and

214 ZNF217, in uninduced and induced DIE and DIE-KO cells. Every sample contains 4 points,

215 representative of the 4 technical replicates. (F) TF perturbations analysis identifying transcription factors

216 that are linked to the i) upregulation and ii) downregulation of the DE genes found in this study, by the 217 activation or inhibition of such TFs. Activation: OE or ACTIVATION, inhibition: KO, KD, SIRNA, SHRNA, 218 INACTIVATION, or INHIBITION. (G) Quintuple Venn diagram comparing DUX4 i) upregulated and ii) 219 downregulated genes found in this study (Ashoti) to those found in previous transcriptomic studies 220 (Geng with P-value $\leq 0.01$, FDR $\leq 0.05$, abs $L 2 F C \geq 1$; Rickard with Padj value of $<0.005$ and abs $L 2 F C$ $221>2$; Jagannathan with P-value $\leq 0.01$, FDR $\leq 0.05$, abs $L 2 F C \geq 1$, Heuvel with P-value $\leq 0.005$, FDR $\leq$ 2220.05 , abs L2FC $\geq 1$ ). 
bioRxiv preprint doi: https://doi.org/10.1101/2020.07.27.223420; this version posted July 27, 2020. The copyright holder for this preprint (which was not certified by peer review) is the author/funder, who has granted bioRxiv a license to display the preprint in perpetuity. It is made available under aCC-BY-NC-ND 4.0 International license.
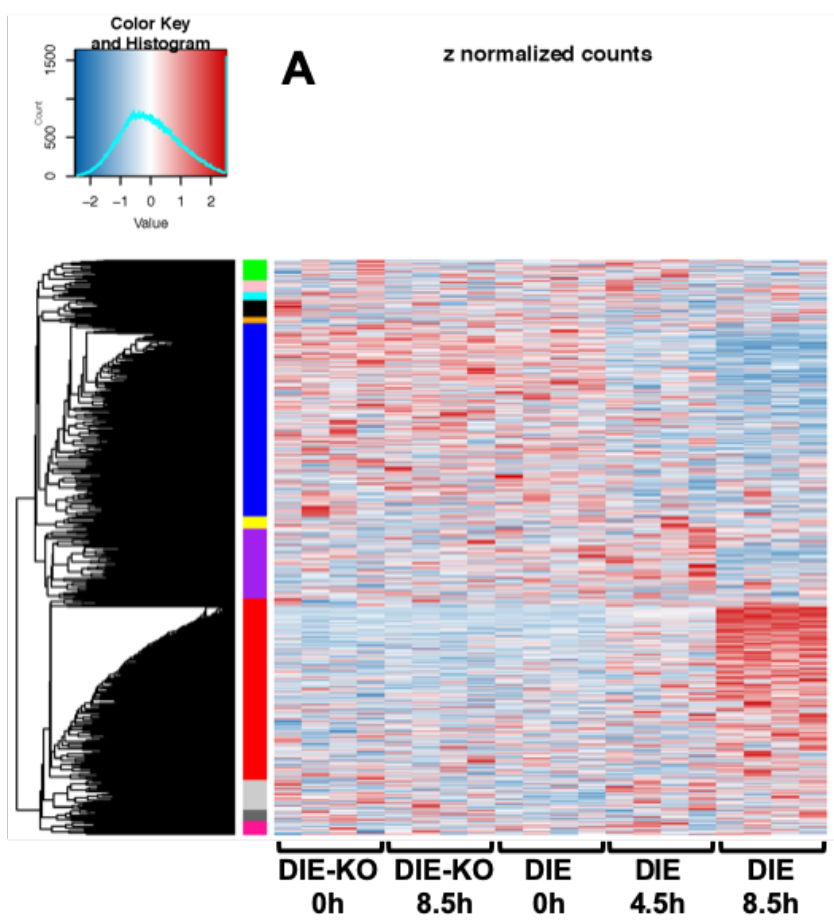

B

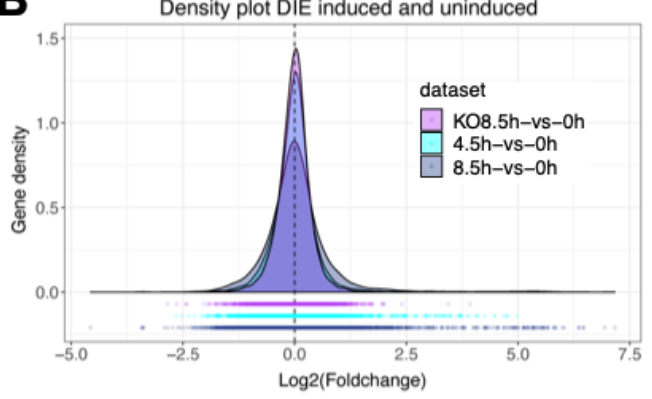

C

Padj $\leq 0.01$, L2FC $\geq 1$

DIE 8.5h N=467

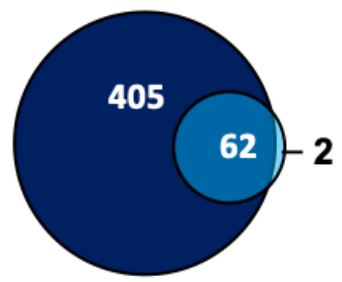

D

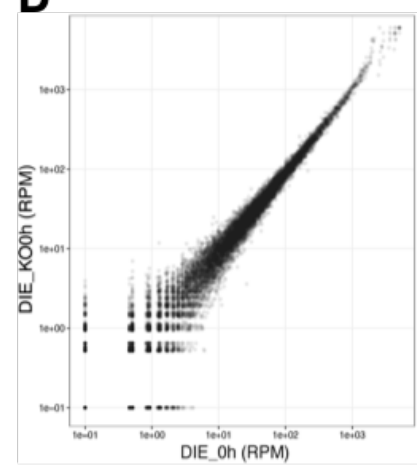

E LEUTX

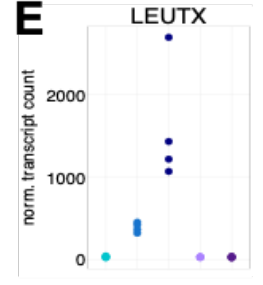

$\mathbf{F}$ DIE 8.5h UP -TF perturbations - expression

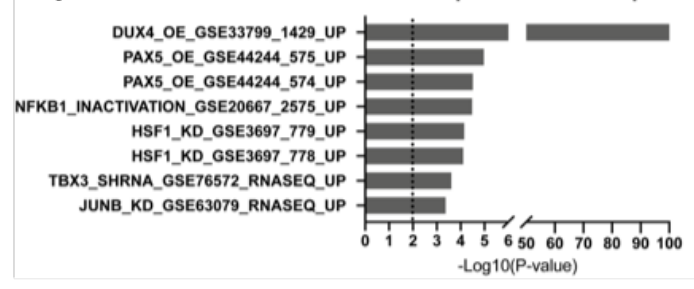

G

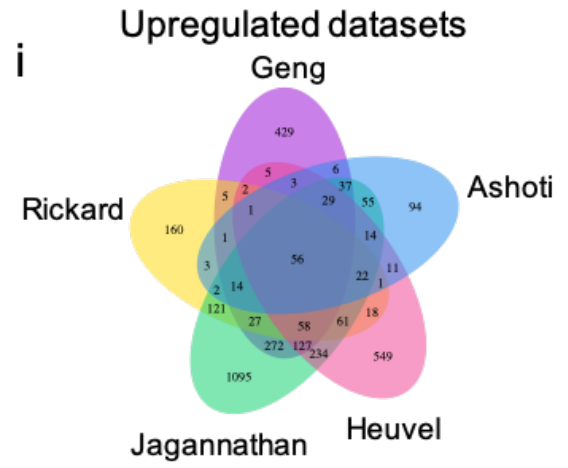

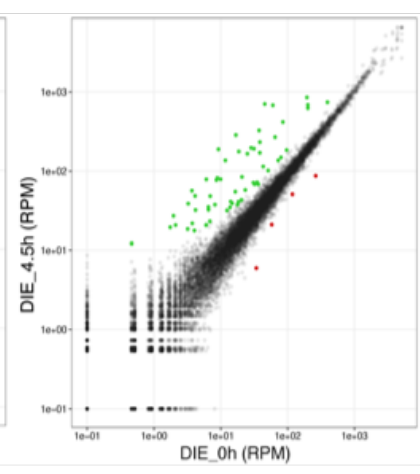
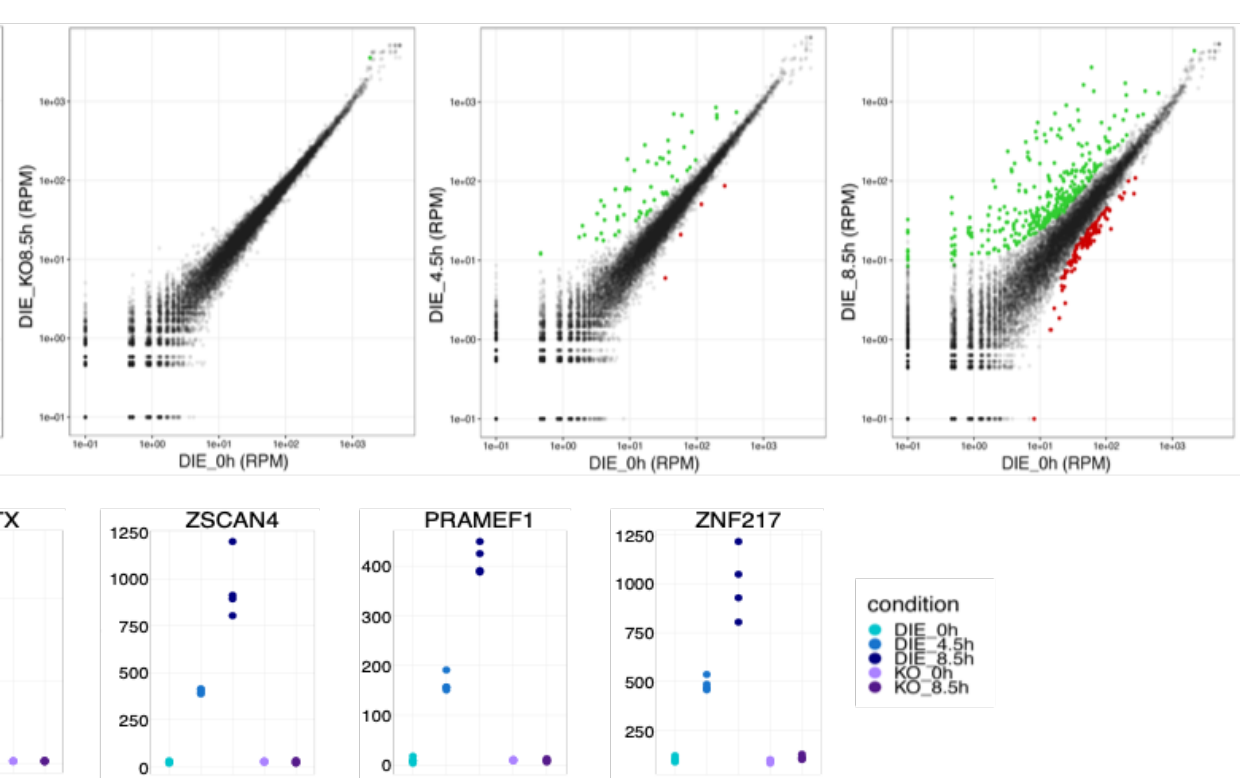

ii

DIE 8.5h DOWN -TF perturbation - expression
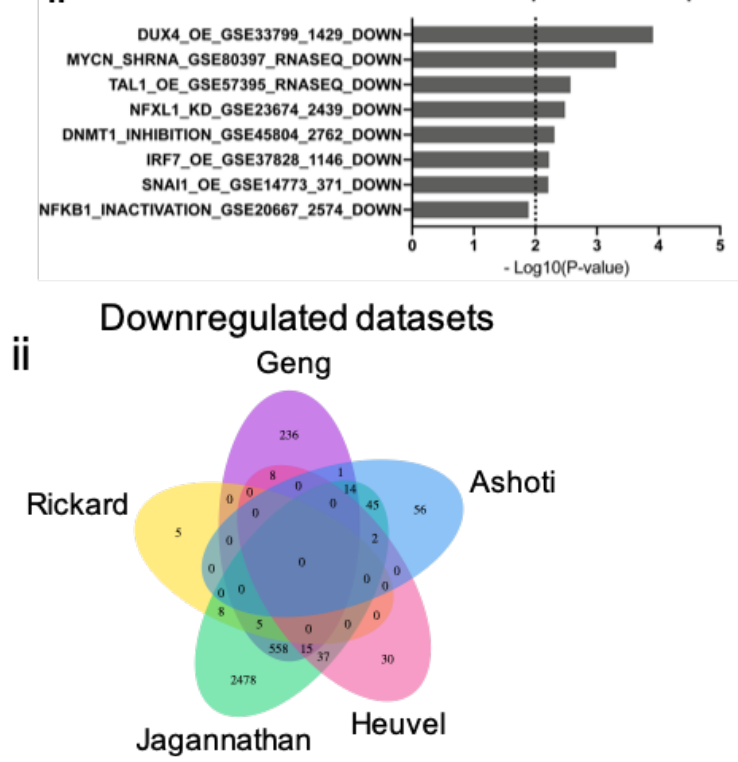

Jagannathan Heuvel 


\section{Genome-wide CRISPR Screen reveals large chromosomal truncations}

226 Using our DIE cell system, we sought out to identify modulators of DUX4 cytotoxicity

227 by performing a genome wide CRISPR/Cas9 knockout screen. The Brunello human

228 CRISPR knockout pooled library was used for this purpose ${ }^{55}$. This library contains

22977.441 gRNAs targeting all protein coding genes, with an average of 4 gRNAs per

230 gene as well as 1000 non-coding control gRNAs. To optimize the signal-to-noise ratio

231 of the experimental system, we titrated the timing and dose of the doxycycline-

232 mediated DUX4 induction and selected two conditions, low (250 $\mathrm{ng} / \mathrm{ml})$ and high

$233(1000 \mathrm{ng} / \mathrm{ml})$ doxycycline with an exposure time of $24 \mathrm{~h}$ (Fig. 3A). At these

234 concentrations 95 to $99 \%$ of the cells die respectively. Figure 3B outlines the setup of

235 the screen. In addition to the high and low doxycycline concentrations, cells were

236 harvested at two timepoints after doxycycline exposure to allow recovery, early (24h)

237 and late $(72 \mathrm{~h})$, ultimately resulting in 4 separate 4 screens; low doxycycline/early

238 harvest, low doxycycline/late harvest, high doxycycline/early harvest, and high

239 doxycycline/late harvest.

240 Upon doxycycline administration and induction of DUX4 expression, cells from the

241 surviving populations were harvested and genomic DNA was sent for sequencing.

242 Sequencing results of the treated samples revealed a large number of significantly

243 enriched hits (Fig. 3C and S3 for all 4 screens), including DUX4 itself and some others

244 performing as well as the DUX4 gRNAs. However, upon closer examination it became

245 clear that the majority of these enriched guides were located on the $q$ arm of

246 chromosome 5, suggesting an FSHD unrelated, experimental artefact. Since the rtTA

247 transgene responsible for DUX4 induction is located on the $5 q$ arm, it is likely that

248 when Cas9 is being targeted to the q-arm of chromosome 5 it leads to the removal of

249 the rtTA transgene, potentially through generation of a large deletion, chromosomal

250 truncation or chromosomal rearrangement. It appears that as the rtTA integration site

251 is located at the end of chromosome $5 q$, each target upstream of this site (towards the

252 centromere) can cause a Cas9-mediated truncation, thereby removing the rtTA. (Fig.

$2533 \mathrm{D}$, for phenograms of all 4 screens see S4). The correlation between the significance

254 of a hit and its position along chromosome 5 highlights the strong association of these

255 unexpected chromosomal rearrangements and the integration of rtTA at the end of 
chromosome 5, where the most significant hits reside in all four screens

257 (Supplementary Figure S5).

259 Some of these $5 q$ locating guides (Fig. 3E) were tested individually in DIE cells 260 containing a constitutively expressing Cas9 its genome (DIE-Cas9), and without 261 selecting for the rtTA and DUX4 transgene. No increased survival was detected 262 compared to the background surviving cells that are seen in the non-target control 263 situation (Fig. 3F). This suggests that the Cas9-induced truncation of a chromosomal 264 arm and subsequent removal of rtTA activity is a rare event that was only identified 265 due to the high sensitivity of our screen.

266 Data shown here was analyzed one-sidedly, and only truly represent enrichment. 267 When analyzing the screen data double sided, one can again notice a clear 268 enrichment of gRNA sequences, however no real depletion (Fig. S6).

A

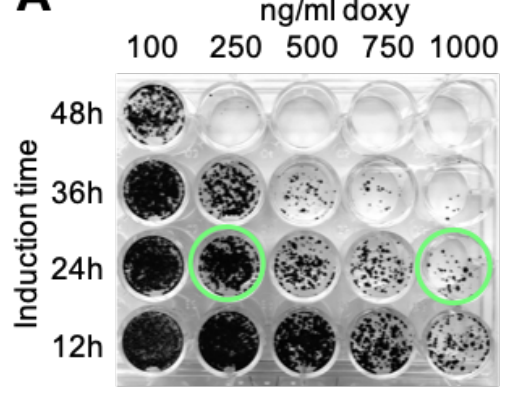

C

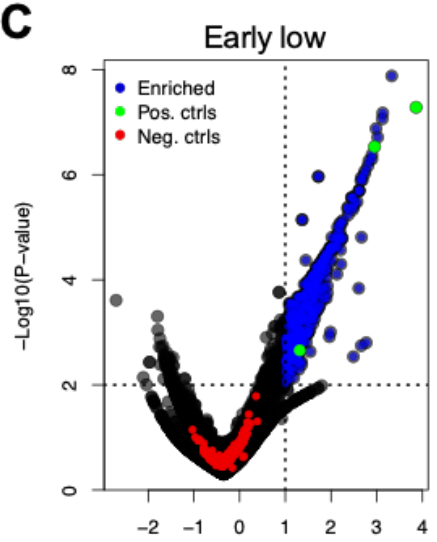

271

$\mathbf{E}$

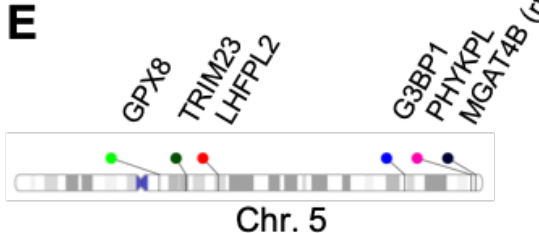

B

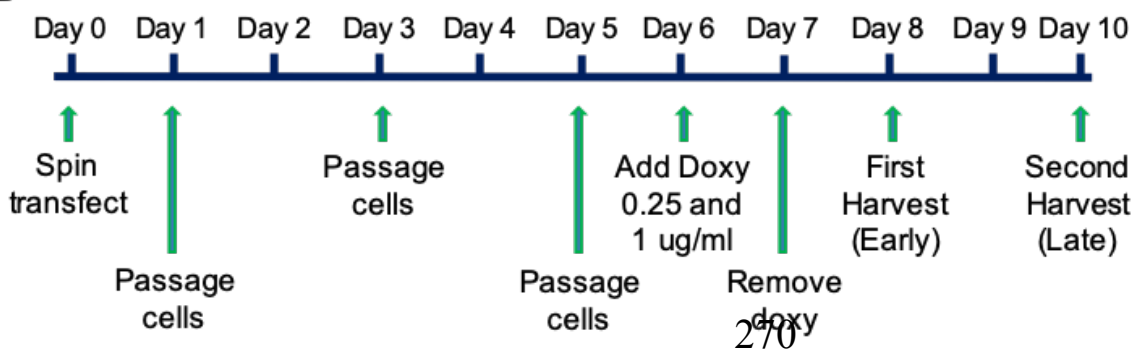

D

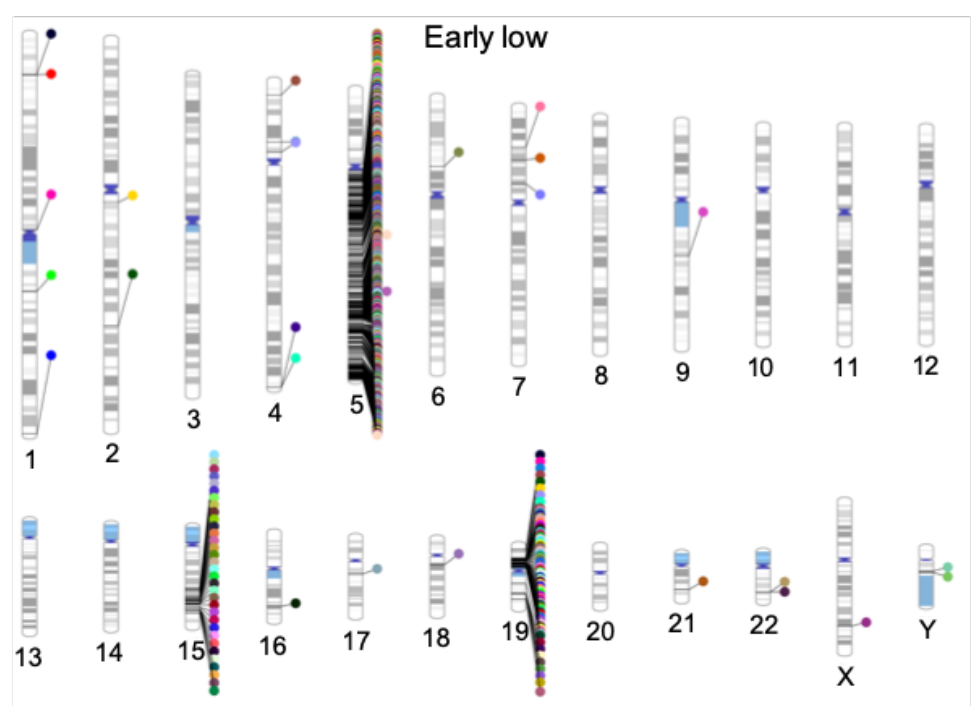

F DUX4 MGAT4B $\quad$ MAST $\quad$ GPX8 $\quad$ TRIM23 LHFPL PHYKPL G3BP1 NT 
Figure 3. CRISPR Screen set up and discovery of a Cas9 artefact. (A) Viability staining of DIE cells treated with doxycycline titration curve to determine which concentration and exposure time to use to induce sufficient cell death rates in DIE cells. Green circles indicate which conditions were used for the genome wide CRIPSR/Cas9 screen. (B) The CRISPR/Cas9 screen timeline from the moment of library transfection (Day 0) to the final harvest of surviving DIE cells (Day 10). (C) Volcano plot showing the enrichment of sets of guides of the low doxycycline-early harvest screen. For a two-sided analysis see supplementary figure S6. Data shown here shows the average log2FC and log10PV of each guide set (set: 4 guides per gene). The Log2(foldchange) is plotted on the X-axis and the -Log10(p-value) on the $\mathrm{Y}$-axis. Blue points represent guide sets that are significantly enriched ( $P$-value $\leq 0.01$ ), $L F C \geq 1$ ), green point are the positive controls (DUX4, MAST1, MGAT4B), red points represent the Non-Target/negative control guides. (D) Chromosomal ideogram indicating the location of enriched hits in the human genome, of the low doxycycline-early harvest screen. (E) Schematic representation of the location of a small number of false positive hits on chromosome 5. (F) Viability staining demonstrating surviving DIECas9 cells containing knock-outs of the same genes mentioned in (C), after 250ng/ml doxycycline exposure. Media did not contain any selection markers. NT: Non-Target controls.

\section{Filtered Genome wide CRISPR screen results reveal no single targetable gene}

Since potential hits were likely obscured by the large number of false-positive hits that resulted from Cas9-mediated elimination of either the DUX4 or the rtTA transgenes, we filtered the screen results to remove all hits located on the q-arm of chromosome 5 , or the p-arm of chromosome 19 (Figure 4A). After analyzing individual guides for their apparent effectiveness in the genome wide screen (instead of the group average), a list of potential hits emerged ( $P \leq 0.05$, Log2foldchange $\geq 1$ ) for each of the 4 screens. Figure 4B shows the number of potential hits that met these criteria for each screen and how many of these hits are shared between them (See Table S4 for the full lists of hits). We further focused on hits that emerged in at least 3 out of the 4 screens. Hits were validated by performing individual knock outs in the DIE-Cas 9 cells, now also containing an inducible eGFP in its genome (DIE-Cas9-ieGFP). The TRE controlling eGFP expression is identical to the TRE controlling DUX4 expression. If there is a true target that can mitigate the apoptotic phenotype without interfering with the inducible system, these positively targeted cells should not only survive but also emit an eGFP signal upon doxycycline admission (Fig. S7). Results show that MED25 increased cell survival when knocked-out (Fig. 4C). MED25 is a subunit of Mediator, a large complex that functions as a bridge between transcription factors and the transcriptional machinery. This includes RNA polymerase II, needed for the 
transcription of all protein coding genes in eukaryotes (reviewed by Soutourina ${ }^{77}$ ). The rescue seen in doxycycline induced DIE cells after MED25 knock-out diminishes upon

309 higher doxycycline exposure, suggesting that loss of MED25 provides a partial rescue.

310 Other genes belonging to the same mediator complex, that initially didn't meet our

311 criteria, were reevaluated by lowering the parameters $(P \leq 0.05$, foldchange of $\geq 1.5)$,

312 identifying a number of other subunits of the mediator complex. When individual

313 knock-outs of these genes were performed, two more subunits of the Mediator-

314 complex showed partial rescue (Fig. 4D). Finally, the individually tested KO cells were

315 analyzed by flow cytometry, for the detection of eGFP. FACS analysis reveals that

316 Mediator-complex components have a general effect on the inducible transcription of

317 DUX4, since the knock-out of Mediator genes also reduced rtTA-inducible eGFP

318 expression, suggesting that their survival was due to a generally reduced ability of

319 rtTA to mediated transgene activation (Fig. 4E).

Figure 4. Filtered CRISPR screen data and validation of potential hits. (A) Adjusted volcano plot of low doxycycline-early harvest screen showing the enrichment of sets of guides targeting genes not located on chromosome $5 q$ or chromosome 19p. Blue points represent guide sets that are significantly enriched ( $P$-value $\leq 0.01$ ), LFC $\geq 1$ ), the green point is the positive control (DUX4), red points represent the Non-Target control guides. (B) Venn diagram showing the overlap of filtered hits between the four screens (EL: Early harvest-Low doxy, LL: Late harvest-Low doxy, EH: Early harvest-High doxy, LH: Late harvest-High doxy). (C) Viability staining showing surviving DIE cells containing single knockouts of potentials hits, identified in the CRISPR screen, after exposure to 3 different concentrations of doxycycline. (D) Viability staining showing the surviving DIE-ieGFP-Cas9 cells containing single knockouts of mediator complex subunits, after exposure to $250 \mathrm{ng} / \mathrm{ml}$ doxycycline. (E) FACs data showing GFP positive cells in surviving populations of DIE-ieGFP-Cas9 cells containing single knockouts. DUX4 knock outed DIE-ieGFP-Cas9 cells comprise $42 \%$ of eGFP positive cells. rtTA, MED25,

333 MED24 and MED16 knock-outs show little eGFP expressing cells, containing between $1.2-4 \%$ of eGFP 334 expressing cells. 
A

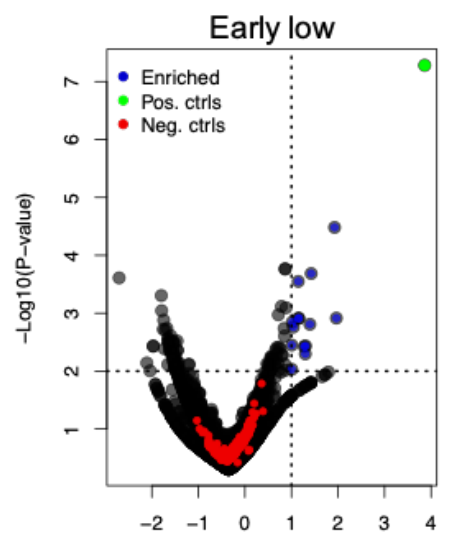

B

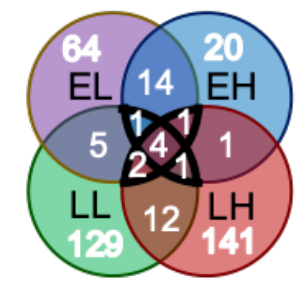

C Log2(fold-change)

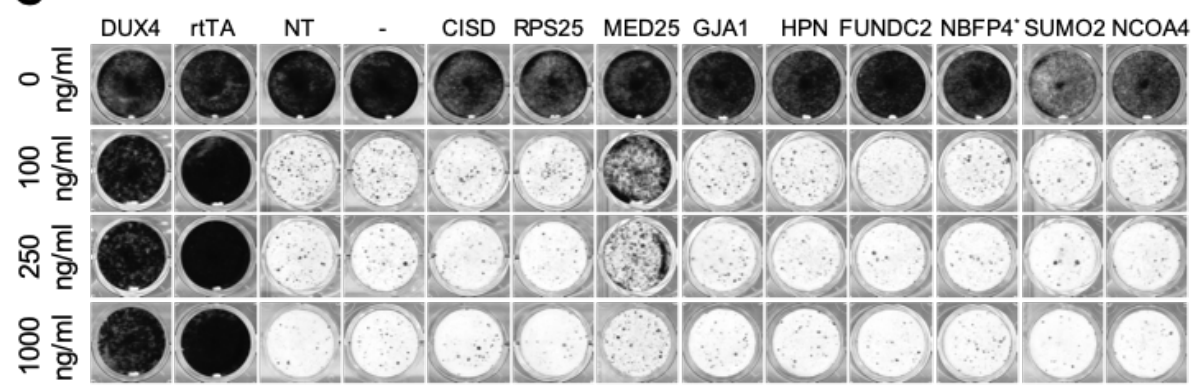

D $250 \mathrm{ng} / \mathrm{ml}$ doxy

DUX MED25 NT CCNC TRIP4 MED7 MED12 MED15 MED16 MED18 MED19 MED24 MED29 MED31 (4)

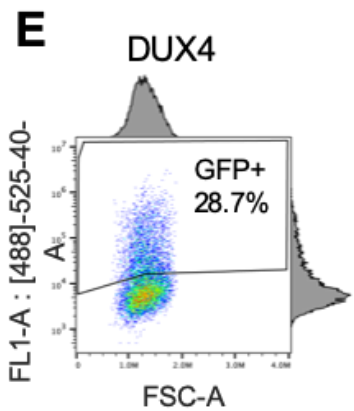

336

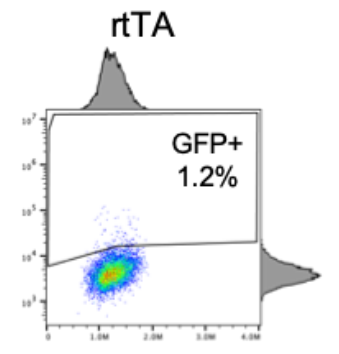

FSC-A

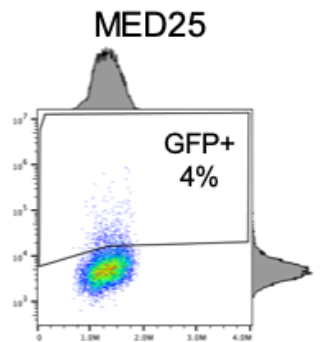

FSC-A

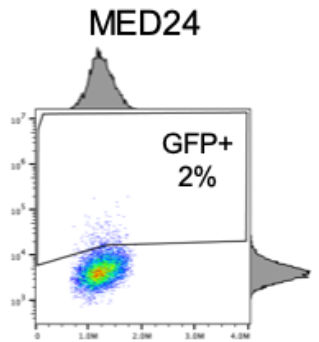

FSC-A

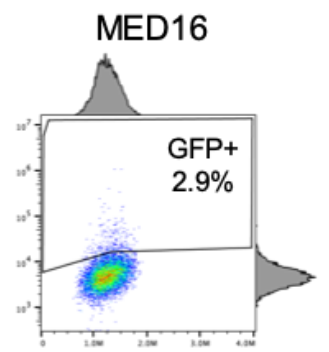

FSC-A

337 In a recent study by Shadle and colleagues, a siRNA screen was performed targeting

338 the "druggable" genome to identify pathways of DUX4 toxicity. The study revealed the

339 MYC-mediated apoptotic pathway and the viral dsRNA-mediated innate immune

340 response pathway to be involved in DUX4 induced apoptosis ${ }^{78}$. We examined our data

341 for enrichment of gRNA sequences that target the genes identified in the Shadle study,

342 but did not observe significant enrichment in our CRIPSR screen data of these

343 sequences. Figure 5A shows data plots that display the enrichment (L2FC) and

344 significance (-Log10(P-value)) of DUX4 and 3 other genes that were initially

345 considered hits, yet later single knock-outs validations demonstrated them to either 
have a generally effect on transcription (MED25), or upon their knockout not exhibiting

347 any additional survival in induced DIE cells (RPS25 and CISD). Genes were only

348 considered if a minimum of one gRNA showed significant enrichment in at least 3 out

349 of 4 screens. Genes involved in the pathways identified by Shadle et all. did not meet

350 these criteria (Fig. 5B). Furthermore, knocking out these genes in the DIE cells did not

351 show an increased survival compared to background noise (Fig. 5C, top panel), as is

352 noticeable in some of the false positives identified during this CRIPSR screen (Fig.

$3535 \mathrm{C}$, lower panel). It should be mentioned that the two screens have major technical

354 differences, such as screening method, complete or partial loss of function, different

355 scale and different cellular backgrounds, most likely all attributing to the little

356 correlation seen between the two studies.

358 Figure 5. Validation of genes involved in the MYC-mediated apoptotic pathway and the viral 359 dsRNA-mediated innate immune response pathway. (A) Data plots showing the significance and 360 enrichment of gRNAs targeting DUX4, MED25, RPS25 and CISD. The Log2(fold-change) of each 361 individual guide is plotted on the left $y$-axis indicated in blue, and the -Log10(P-value) is plotted on the 362 right $y$-axis, in red. When guides fall above the blue and red intermitted ablines, they are considered 363 significant (Log2(foldchange) $>1$, -Log10(P-value) $>1.3$ ). The gRNAs that are significantly enriched in 364 all 4 screens are underlined. All 4 gRNAs targeting DUX4 are significantly enriched. 3 out of 4 gRNA's 365 targeting MED25 are significantly enriched (guides 1, 2 and 3). Guides 1 and 4 targeting PRS25 are 366 significantly enriched, and CISD has one guide that is significantly enriched in all 4 screens. (B) Data 367 plots showing the enrichment of gRNA targeting FOSB, RNASEL, MYC, FXN and EAF1. None of the 4 368 guides show significant enrichment in any of the 4 screens. (C) Viability staining showing surviving DIE369 Cas9 cells containing single knockouts of genes involved in the MYC-mediated apoptotic pathway and 370 the dsRNA-mediated immune response pathway (Top panel). Controls can be found in the bottom panel 371 and are as followed, positive controls: DUX4, rtTA, MED24, MED16 and MED25; Negative non-target 372 control: NT. 
A
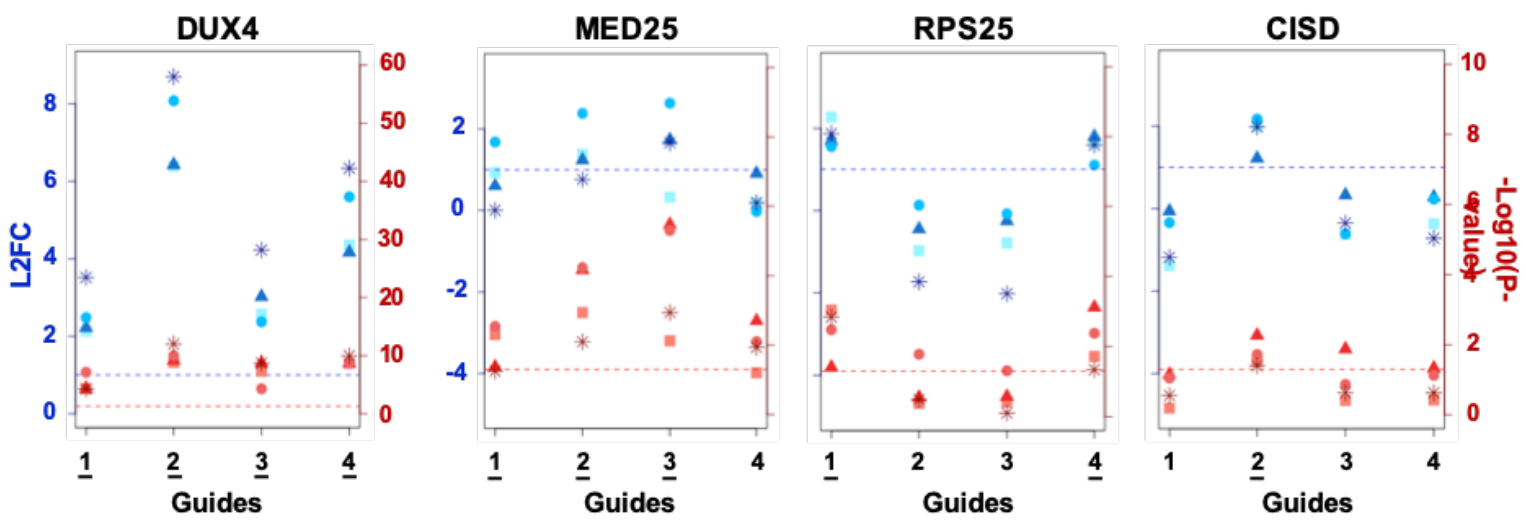

B
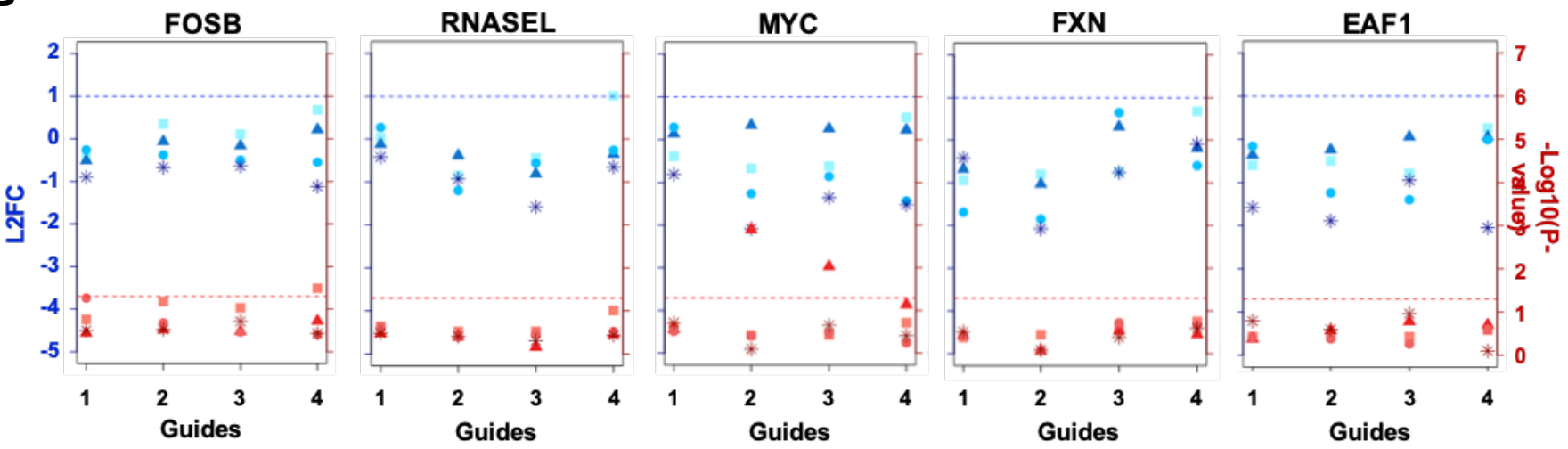

C

FOSB RNASE MYC FXN EAF1 PHF2 NLGN TGS1 HSD17B ITPK1 POMK CDC20 QSOK1 THOP1 FBN2 APH1A

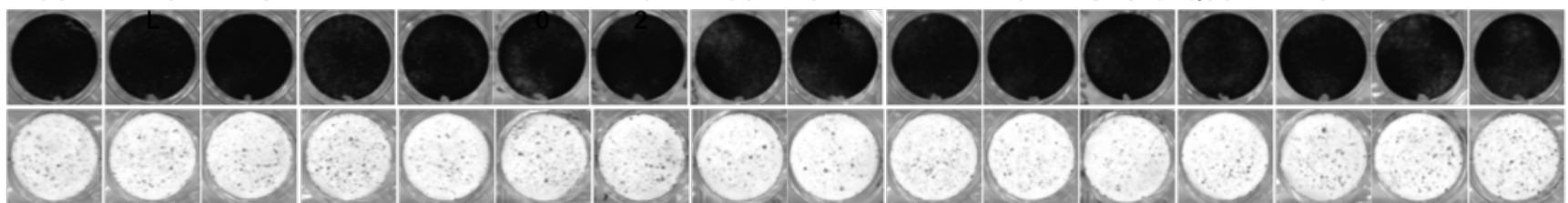

376

377

378

379

DUX4 ITTA MED24 MED16 MED25 NT

380

381

382 Discussion

383 At present there are no effective pharmacological treatment options that can improve

384 muscle strength or slow down disease progression in FSHD patients ${ }^{79}$. Unravelling

385 the underlying mechanism of DUX4 cytotoxicity would help identify therapeutic targets.

386 We hypothesized that inhibition of key downstream DUX4 effectors would slow or

387 abrogate the cytotoxic process, and set out to identify such genes by performing an

388 exhaustive genome wide CRISPR/Cas9 screen. We know the screen was exhaustive 
because we picked up rare rearrangements disabling the DUX4 transgene or the rtTA inducer. The goal of the screen was to identify targets that can mitigate DUX4 induced toxicity. While the screen's technical execution went very well and displayed high sensitivity and specificity toward candidate editing events that indeed mitigated cytotoxicity in our transgene model, none of the obtained hits had a direct effect on DUX4 or its downstream transcriptional network. Rather, screen hits seemed to specifically affect the experimental system itself, either by affecting the tetracyclineinducible system responsible for DUX4 transgene induction, or by mutations of the DUX4 transgene. The main contributor of the false positive result is likely a rare Cas9induced chromosomal truncation event, that removes the transgenes when targeted to the chromosomal arm to which they have integrated. Although these events appear to be rare, nearly all guides that targeted genes located on the chromosomal arm to which rtTA had integrated $(5 q)$ were robustly enriched, underwriting the sensitivity of this screening method. Most remaining hits did not appear to effect DIE cell survival upon individual validation, but members of the Mediator complex did show a positive effect on survival. Unfortunately, these mediator subunit genes seemed to generally suppress rtTA-mediated transcription so their mitigating effect was not mediated by specifically altering DUX4 cytotoxicity. We therefore concluded, that based on the conditions used in this study, there to be no individual target (other than DUX4 itself) that upon knockout, can provide a strong inhibition of DUX4 induced cytotoxicity. We therefore believ that efforts should therefore be redirected to the direct modulation of DUX4.

411 While our library only targeted protein-coding genes, we believe we would have 412 picked-up any mitigating non-coding RNAs as well, had they provided a strong rescue 413 from the DUX4 cytotoxic effects. In that case, one would have expected to see a 414 similar hotspot of gRNAs on and around the true target sites, as we observed for, 415 MED16, where a hotspot of gRNAs was observed on the p-arm of chromosome 19, corresponding to the location of MED16. Another hotspot can be seen on the q arm of chromosome 19, corresponding to the location of MED25. The hotspot on chromosome 15 can be explained by the genetic makeup of the HAP1 cell line. HAP1 cells not only have the Philadelphia chromosome, but also an integration event where a region of chromosome 15 integrated on chromosome $19 \mathrm{p}^{80}$. The hotspot on 
421 chromosome 15 correlates to the region that has integrated on chromosome 19p,

422 close to the MED25 site.

423 Our screen results shown here do not corroborate previous findings of Shadle et al78.

424 However, their siRNA screen differs in many aspects to the performed genome wide

425 CRIPSR/Cas9 screen we executed. Their screen was knocking-down the druggable

426 genome, using Lipofectamine RNAiMAX to deliver the siRNA library, in

427 Rhabdomyosarcoma derived cells; whereas our screen was knocking-out protein

428 coding genes genome wide, using a viral library, in chronic myeloid leukemia derived

429 cells. These differences could explain why results between the two screens are not

430 correlating with one another. Furthermore, A side by side comparison study of

431 CRISPR/Cas9 and a next generation RNAi screens reveals that the screening

432 methods seem to effect different biological aspect of the cells, therefore finding little

433 correlations between results. The authors also in part attribute these differences to the

434 technical differences between the two techniques ${ }^{37}$.

435 Another recently published genome wide CRISPR/Cas9 study, where a similar

436 methodology was used in a DUX4 inducible, immortalized myoblast line, identified the

437 HIF1 oxidative stress pathway as a modulator of DUX4-induced apoptosis ${ }^{81}$. This

438 data, as well as previous reports clearly demonstrate the role of the HIF1 hypoxia

439 pathway in DUX4-mediated cytotoxicity ${ }^{81-83}$. The HIF1 pathways did not come up in

440 our screen (Fig. S8). This demonstrates that changes in this pathway are likely not the

441 only DUX4 induced cellular changes that push cells towards apoptosis. The fact that

442 the HIF1 pathway did not come up in our screen could also indicate differences in

443 sensitivity to oxidative stress between cellular systems. Different cell types experience

444 and respond differently to oxidative stress, with differences in culturing conditions as

445 a further attributing factor, such as the concentration of 2-mercapto ethanol to cell

446 culture media.

448 While our screen did not identify target genes that can mitigate DUX4 cytotoxicity, it 449 does illustrate some important aspects that need to be considered when performing 450 phenotypic CRISPR/Cas9 screens. One being the likely large chromosomal 451 truncations that can be induced Cas9, a phenomena also recently reported by Cullot 452 et $\mathrm{al}^{84}$. While these are rare events in a cell population, our results demonstrate that 
453

454

455

456

457

458

459

460

461

462

463

464

465

466

467

468

469

470

471

472

473

474

475

476

477

478

479

480

481

482

483

484

in a sufficiently sensitive screening system, they are robustly identified and can crowd potential positive hits. Sufficient selection should at least help in this aspect by removing cells that had their resistance marker (linked to the transgene) deleted. Another aspect that needs consideration are the endogenous genes that have a general effect on transcription and translation, in this case effecting the inducible system, like subunits of the mediator complex identified in this study. Potential hits will always need to be validated individually in such a way that can exclude this possibility, like shown here, or by Shadle et al. where some of the same genes were identified effecting their inducible Tet-On system ${ }^{78}$.

This study started out with the aim of trying to contribute to the understanding of the underlying molecular mechanism of FSHD, by performing a genome wide CRISPRCas9 phenotypic screen. However, with no significant hits that can explain their contribution to the apoptotic phenotype, this story also tells a cautionary tale for knockout screens through the use CRISPR-Cas9, which can benefit future groups planning to execute similar screens.

\section{Methods}

\section{Cloning and generating the DIE cell line}

To generate the inducible DsRed/DUX4 system, the third generation lenti-viral plasmid pRRLsincPPT-wpre ${ }^{85}$ was used as the backbone. The linearized viral backbone was created by restriction digestion using the following enzymes: Hpal and Sall (NEB). All inserts were generated with PCR amplification using phusion DNA polymerase (Fischer Scientific). Insert were created with 15bp adapter sequences, matching the backbone or neighboring fragments, for in-fusion cloning (Clontech). The first fragment consisted of CPPT/CTS-TRE-mCMV sequences, and the second fragment contained the LoxP-DsRed-LoxP (LSL) sequence. After inserting these two fragments into the pRRLsincPPT-wpre backbone, this newly cloned construct was transformed into chemically competent Stbl3 Escherichia coli (E.coli). The plasmid was isolated and purified from the Stbl3 cells using the HiPure plasmid kits from Invitrogen (Fischer 
scientific). This TRE-LSL plasmid was then opened up using restriction enzymes Xbal and Ecrol (NEB). After which the remaining three inserts: DUX4(exon1-3), mPGK and PuroR-WPRE, were cloned downstream from the LoxP-DsRed-LoxP in similar fashion.

The DIE cell line was obtained by transducing diploid HAP1 (DIPH) cells with lentiviral particles containing the inducible DsRed/DUX4 cassette mentioned above. 2 days after lentiviral transduction, positively transfected cells were selected with puromycin. After establishing a stable line by puromycin selection, lentiviral particles containing CMV-rtTA3-BlastR were added to the DsRed/DUX4 containing DIPH cells. Positively transfected cells were subsequently selected with basticidin, and FACs sorted for DsRed expression upon exposure to doxycycline. The pLenti CMV rtTA3 Blast (w7561) was a gift from Eric Campeau (Addgene plasmid \#26429).

\section{Cell culture}

500 DIPH cells were cultured in IMDM media (Fischer Scientific) supplemented with 10\% FBS. The DIE cells were cultured in IMDM media supplemented with $10 \%$ Tet system

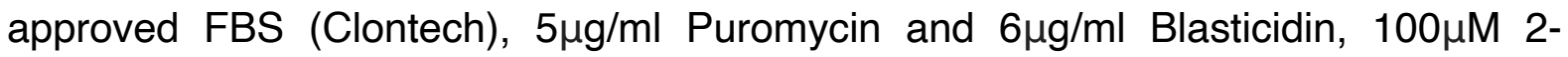
mercaptoethanol.

Cloning p2T-Cas9, p2T-ieGFP and sgRNA constructs, and generating DIE-Cas9 and DIE-Cas9-ieGFP cell lines

The p2T-CAG-spCas9-NeoR mammalian expression plasmid was created by replacing the Blasticidin resistance gene (BlastR) in the p2T-CAG-spCas9-BlastR (Addgene: 107190) 86 with a Neomycin resistance gene (NeoR). The p2T-CAGspCas9-BlastR plasmid is contained in a p2Tol2 backbone ${ }^{87}$. The BlastR gene was removed using restriction digestion, using Mfel and AfIII (NEB). Cloning the NeoR DNA

512 fragment into the p2T-CAG-spCas9 backbone was done in similar fashion as described above. The p2T-CAG-SpCas9-BlastR was a gift from Richard Sherwood.

514 The p2T-TetO-eGFP-HygroR plasmid was generated in a similar way as the p2T-

515 CAG-spCas9-NeoR. In short, all sequences between transposable elements of a p2T 516 plasmid were removed by restriction digestion using Alel and EcoRI (NEB). The TetO- 
517 eGFP-HygroR cassette was created by amplifying each subunit individually from

518 already excising constructs, and thereafter cloned into the empty p2T backbone, using

519 in-fusion cloning.

520 Both p2T-CAG-spCas9-NeoR and p2T-TetO-eGFP-HygroR were introduced in the

521 DIE cell line by using Transposase. The p2T-CAG-spCas9-NeoR was introduced into

522 DIE cells together with a plasmid encoding for transposase, using Polyethylenimine

523 (PEI) transfection reagent (4ug PEI per 1ug DNA). The DIE cells were exposed to the

524 transfection mixture for 14-16h, after which the transfection media was replaced with

525 growth media. Geneticin g418 selection was started two days post transfection,

526 generating the DIE-Cas9 line. The DIE-Cas9-ieGFP cell line was created by adding

527 Transposase and p2T-TetO-eGFP-HygroR the DIE-Cas9 line, described as above.

528 spCas9-sgRNA constructs were cloned using a plasmid containing a U6 promotor, 2

$529 \mathrm{BsmBI}$ sites with directly adjacent the tracrRNA sequence, and a Hygromycin

530 resistance gene (made in house). This U6-2xBsmBI-Tracr-HygroR plasmid was

531 digested with the BsmBI restriction enzymes (NEB), after which the CRISPR inserts

532 were ligated in using T4 DNA ligase (NEB). CRISPR inserts were generated by

533 annealing two complementary oligos containing a $4 \mathrm{bp}$ adapter serving as the $\mathrm{BsmBI}$

534 sticky end.

535 All plasmids mentioned in this study were transformed in chemically competent Stbl3

536 Escherichia coli (E.coli), and prepped using a HiPure plasmid Midi or Maxi kit

537 (Invitrogen).

538

$R N A$ extraction and $R T-q P C R$

540 Cultured cells were rinsed with DPBS just prior to the additional of TRIzol reagent

541 (Thermo Scientific). Total RNA samples were subsequently extracted by addition

542 chloroform, and isopropanol precipitation, and finally treated with RNase free DNase

543 I (Promega). Reverse transcription was performed using the Superscript III kit

544 (Invitrogen) and random primers (Promega), generating cDNA. Quantitative PCR was

545 then initiated using IQ SYBR Green Supermix (Bio-Rad 1708880), 50 ng of cDNA, and

546 the following gene-specific primers: 
- ZSCAN4: 5'-GTGGCCACTGCAATGACAA-3', 5'-AGCTTCCTGTCCCTGCATGT-3'88;

- ZNF217: 5'-AAGCCCTATGGTGGCTCC-3', 5'-TTGATATGACACAGGCCTTTTTC-388';

- PRAMEF1: 5'-CTCCAAGGACGGTTAGTTGC-3', 5'-AGTTCTCCAAGGGGTTCTGG-388';

- LEUTX: 5'- GGCCACGCACAAGATTTCTC-3', 5'- TCTTGAACCAGATCTTTACTACGGA-3';

- rtTA: 5'- CCCTGCCAATCGAGATGC-3',

\section{Protein extraction and Western blot}

DIE cells were harvested by trypsinization and lysed with RIPA buffer. Total protein concentrations were determined using a Pierce BCA protein assay kit (Fischer Scientific). 20ug protein was denatured using $4 x$ Laemmli sample buffer (Bio-rad) with 10\% BME (Sigma), and boiled for 5 minutes. Samples were run on a $15 \%$ SDSpolyacrylamide gel and transferred to a PVDF membrane (Merck). Membranes were blocked for an hour using 5\% BSA in TBST, and were subsequently incubated overnight with anti-DUX4 antibody [E5-5] (Abcam, ab124699) in blocking solution (5\% BSA in TBST), at $4^{\circ} \mathrm{C}$. Membranes were than incubated for an hour with Secondary goat anti-rabbit-HRP antibody (Santa Cruz, sc-2004), and primary rabbit mAb $\beta$-Actin HRP conjugated antibody (Cell signaling, 5125s) in blocking buffer. Chemiluminescent signal was detected using GE ImageQuant LAS 4000 imager, upon admission of Pierce ECL Plus Western Blotting substrate (Fischer Scientific). 
RNAseq sample preparation and sequencing

583 Cultured cells were rinsed with DPBS just prior to the additional of TRIzol reagent 584 (Thermo Scientific). Total RNA samples were subsequently extracted by addition chloroform, and isopropanol precipitation. The library prep was performed using CELseq1 primers $^{91}$ and the Life technologies Ambion kit (AM1751) ${ }^{92}$, and were processed using CEL-seq2 protocol93. Samples were sequenced using Illumina Nextseq 500, 2x75 kit, high output. Four technical replicates per samples were send for sequencing, and were sequenced to an average of 600.000 reads per replicate (combined read count of 2.4 million reads per sample). Differential expression analysis was done using the DESeq2 package ${ }^{94}$.

\section{Doxycycline titration curve}

594200.000 cells were seeded into wells of a 24-wells plate and incubated overnight at $5 \% \mathrm{CO}_{2}$, and $37^{\circ} \mathrm{C}$. When cells reached a density of $90-100 \%$ confluency, different concentrations of doxycycline were added to the vertical lanes $(100 \mathrm{ng} / \mathrm{ml}, 250 \mathrm{ng} / \mathrm{ml}$, $500 \mathrm{ng} / \mathrm{ml}, 750 \mathrm{ng} / \mathrm{ml}, 1000 \mathrm{ng} / \mathrm{ml}$ ), with the horizontal lanes experiencing different exposure times (48h, 36h, 24h, 12h). After a recovery period of 96h (after doxy exposure was ended), cells were washed with DPBS, and fixed with Methanol for 10 minutes. Giemsa stain, modified solution (Sigma) was subsequently added for 45 minutes, after which it was removed and the wells were washed with demineralized water.

\section{Genome-wide CRISPR screen}

605 The screen on the DIE line was performed as previously described by Doench et al. ${ }^{55}$ and Sanson et al. ${ }^{95}$. Due to a shared selection marker between the DIE line and the all-in-one Brunello lentiviral library, transfected cells could not be selected for, thus the total number of cells was raised to 1500 cells per guide, when considering an average transfection efficiency of $30-50 \%$ in all cell lines tested by Doench et al ${ }^{55}$. To minimize the probability of multiple sgRNA plasmids entering one cell, we determined the

611 transfection efficiency and calculated the MOI. With 1500 cells per guide (total of 61277.441 guides), each of the three replicates contained $120 * 10 \mathrm{E} 6$ cells. These cells 
613 were spin transfected for $2 \mathrm{~h}$ at $1000 \mathrm{~g}$ with $82^{*} 10 \mathrm{E} 6$ Brunello virus particles

614 (LentiCRISPRv2, Addgene 73179-LV, all-in-one system in which every plasmid

615 contains SpCas9, and a guide RNA) reaching a $\mathrm{MOI}$ of 0.65 , and a transfection

616 efficiency of around $60 \%$ upon testing the viral library on the DIPH parental line. After

617 transfection the $120 * 10 \mathrm{E} 6$ transfected cells (contained in 40 wells of 12-well tissue

618 culturing plates) were trypsinized and passaged to $60145 \mathrm{~mm}$ TC plates. Mutagenized

619 cells were maintained for 6 days, before inducing a set of 24 plates with either a low

620 or high doxycycline concentration (low: 250ng/ml, high: $1000 \mathrm{ng} / \mathrm{ml}$ ). The remaining 12

621 plates were harvested for cryofreezing (7 plates) and for determining library coverage

622 (5 plates). After a $24 \mathrm{~h}$ doxycycline induction period, 12 plates were given a $24 \mathrm{~h}$

623 recovery period (early harvest) of both the low and high doxycycline exposed sets.

624 The remaining 24 plates received an additional $48 \mathrm{~h}$ of recovery time (late harvest),

625 before harvesting the surviving cells for sequencing (Fig. S1). Cell Pellets were stored

626 at $-80^{\circ} \mathrm{C}$ until further processing. The Human Brunello CRISPR knockout pooled

627 lentiviral prep library was a gift from David Root and John Doench.

\section{Library prep, sequencing and analysis}

630 Genomic DNA (gDNA) was isolated using NucleoSpin Blood Mini (less than 5 million cells), Midi (L) (5-20 million cells) and Maxi (XL) (more than 20 million cells) kits, depending on the size of cell pellet. Libraries were prepared and sequenced on a HiSeq2000 (Illumina) as described by Doench et al. Analysis was conducted using "STARS", gene-ranking method to generate FDR values developed by Doench et al. that was used to generate $p$-values and FDR rates. ${ }^{55}$ Chromosomal ideogram were generated by using the PhenoGram webtool from the Ritchie lab from the university of Pennsylvania ${ }^{96}$.

640 DIE-Cas9 and DIE-Cas9-ieGFP were seeded in a 24-well setting. Next day, when the 641 cells had reached $70-90 \%$ confluency, cells were transfected with 500ng guide 642 plasmid per well using 4ug PEI per 1ug DNA. During the overnight transfection no 643 selection markers were presents in the media, however growth media was supplemented with $100 \mathrm{U} / \mathrm{ml}$ pen-strep. Cells were passaged with or without selection 
markers during a period of 6-7, after which doxycycline was added (100, 250 or 1000

$646 \mathrm{ng} / \mathrm{ml}$ ) for a $24 \mathrm{~h}$ period. Wells were washed with DPBS to remove dead cells and 647 debris. Remaining cells were given the opportunity to grow out, or to perish (if they

648 had already entered the apoptotic pathway) for an additional 48-96 hours. The wells

649 were stained using Giemsa modified solution, as described previously.

651 Flowcytometry sorting (FACS) and analysis

652 DIE-Cas9-ieGFP cells were induced with $250 \mathrm{ng} / \mathrm{ml}$ doxycycline $24 \mathrm{~h}$ prior to FACS 653 analysis. After the $24 \mathrm{~h}$ doxycycline exposure, cells were trypsinized using $0.25 \%$ 654 Trypsin-EDTA, resuspended in iMDM media supplemented with Tet approved FBS 655 and DAPI nuclear staining, and strained using a Cell-strainer capped tubes (Falcon).

656 Cells were analyzed using the Beckman coulter Cytoflex S flow cytometer.

\section{Imaging DIE cells}

659 Untreated and doxycyline treated DIE cells were stained with AnnexinV-Alexa Fluor 660488 and PI (Thermo Scientific), by adding the staining solutions directly to growth 661 media at a 1:50 and 1:100 ratio respectively. Cells were left to incubate overnight in 662 growth media containing staining solution(s) and with or without doxycycline 663 treatment. Live imaging during treatment of these cells was carried out using a 664 Confocal microscopy (Zeiss LSM 700). Still images were taken after the overnight 665 incubation with am EVOS Digital Color Fluorescence Miscroscope (Invitrogen).

\section{Data Resources}

668 Data containing the bulk RNA sequencing samples in quadruplicate are available 669 from the GEO data base, accession number: GSE154649.

670 Data containing the Genome wide CRISPR/Cas9 samples in triplicate are available 671 from the GEO data base, accession number: GSE155034. 


\section{References}

675 1. Deenen, J. C. W. et al. Population-based incidence and prevalence of 676 facioscapulohumeral dystrophy. Neurology 83, 1056-1059 (2014).

677 2. Tawil, R. \& Van Der Maarel, S. M. Facioscapulohumeral muscular dystrophy. Muscle and Nerve 34, 1-15 (2006).

3. Pastorello, E., Cao, M. \& Trevisan, C. P. Atypical onset in a series of 122 cases with FacioScapuloHumeral Muscular Dystrophy. Clin. Neurol. Neurosurg. 114, 230-234 (2012).

4. Dixit, M. et al. DUX4, a candidate gene of facioscapulohumeral muscular dystrophy, encodes a transcriptional activator of PITX1. Proc. Natl. Acad. Sci. U. S. A. 104, 18157-18162 (2007).

5. Lemmers, R. J. L. F. et al. A unifying genetic model for facioscapulohumeral muscular dystrophy. Science (80-. ). 329, 1650-1653 (2010).

6. Snider, L. et al. Facioscapulohumeral dystrophy: Incomplete suppression of a retrotransposed gene. PLoS Genet. 6, 1-14 (2010).

7. Whiddon, J. L., Langford, A. T., Wong, C. J., Zhong, J. W. \& Tapscott, S. J. Conservation and innovation in the DUX4-family gene network. Nat. Genet. 49, 935-940 (2017).

8. Hendrickson, P. G. et al. Conserved roles of mouse DUX and human DUX4 in activating cleavage-stage genes and MERVL/HERVL retrotransposons. Nat. Genet. 49, 925-934 (2017).

9. Das, S. \& Chadwick, B. P. Influence of repressive histone and DNA methylation upon D4Z4 transcription in non-myogenic cells. PLoS One 11, 126 (2016).

10. Geng, L. N. et al. DUX4 Activates Germline Genes, Retroelements, and

11. Dmitriev, P. et al. DUX4-induced constitutive DNA damage and oxidative Immune Mediators: Implications for Facioscapulohumeral Dystrophy. Dev. Cell stress contribute to aberrant differentiation of myoblasts from FSHD patients. Free Radic. Biol. Med. 99, 244-258 (2016).

12. laco, A. De et al. A family of double-homeodomain transcription factors 
regulates zygotic genome activation in placental mammals. Nat. Genet. 49, 941-945 (2017).

13. Knopp, P. et al. DUX4 induces a transcriptome more characteristic of a lessdifferentiated cell state and inhibits myogenesis. J. Cell Sci. 129, 3816-3831 (2016).

14. Bosnakovski, D. et al. High-throughput screening identifies inhibitors of DUX4induced myoblast toxicity. Skelet. Muscle 4, 1-11 (2014).

15. Bosnakovski, D. et al. DUX4c, an FSHD candidate gene, interferes with myogenic regulators and abolishes myoblast differentiation. Exp. Neurol. 214, 87-96 (2008).

16. Winokur, S. T. et al. Expression profiling of FSHD muscle supports a defect in specific stages of myogenic differentiation. Hum. Mol. Genet. 12, 2895-2907 (2003).

17. Bosnakovski, D. et al. An isogenetic myoblast expression screen identifies DUX4-mediated FSHD-associated molecular pathologies. EMBO J. 27, 27662779 (2008).

18. Bosnakovski, D. et al. p53-independent DUX4 pathology in cell and animal models of facioscapulohumeral muscular dystrophy. DMM Dis. Model. Mech. 10, 1211-1216 (2017).

19. Feng, Q. et al. A feedback loop between nonsense-mediated decay and the retrogene DUX4 in facioscapulohumeral muscular dystrophy. Elife 2015, 1-13 (2015).

20. Rickard, A. M., Petek, L. M. \& Miller, D. G. Endogenous DUX4 expression in FSHD myotubes is sufficient to cause cell death and disrupts RNA splicing and cell migration pathways. Hum. Mol. Genet. 24, 5901-5914 (2015).

21. Jagannathan, S. et al. Model systems of DUX4 expression recapitulate the transcriptional profile of FSHD cells. Hum. Mol. Genet. 25, ddw271 (2016).

22. Van Den Heuvel, A. et al. Single-cell RNA sequencing in facioscapulohumeral muscular dystrophy disease etiology and development. Hum. Mol. Genet. 28, 1064-1075 (2019).

23. Barrangou, R. et al. CRISPR provides acquired resistance against viruses in prokaryotes. Sciencee 315, 1709-1712 (2007). 
24. Brouns, S. J. et al. Small CRISPR RNAs guide antiviral defense in prokaryotes. Science (80-. ). 321, 960-964 (2008).

25. Christian, M. et al. Targeting DNA double-strand breaks with TAL effector nucleases. Genetics 186, 756-761 (2010).

26. Li, T. et al. TAL nucleases (TALNs): Hybrid proteins composed of TAL effectors and FokI DNA-cleavage domain. Nucleic Acids Res. 39, 359-372 (2011).

27. Mussolino, C. et al. A novel TALE nuclease scaffold enables high genome editing activity in combination with low toxicity. Nucleic Acids Res. 39, 92839293 (2011).

28. Miller, J. C. et al. A TALE nuclease architecture for efficient genome editing. Nat. Biotechnol. 29, 143-150 (2011).

29. Kim, Y. G., Cha, J. \& Chandrasegaran, S. Hybrid restriction enzymes: Zinc finger fusions to Fok I cleavage domain. Proc. Natl. Acad. Sci. U. S. A. 93, 1156-1160 (1996).

30. Kim, Y. G., Kim, P. S., Herbert, A. \& Rich, A. Construction of a Z-DNA-specific restriction endonuclease. Proc. Natl. Acad. Sci. U. S. A. 94, 12875-12879 (1997).

31. Smith, J. et al. Requirements for double-strand cleavage by chimeric restriction enzymes with zinc finger DNA-recognition domains. Nucleic Acids Res. 28, 3361-3369 (2000).

32. Bibikova, M. et al. Stimulation of Homologous Recombination through Targeted Cleavage by Chimeric Nucleases. Mol. Cell. Biol. 21, 289-297 (2001).

33. Bibikova, M., Golic, M., Golic, K. G. \& Carroll, D. Targeted Chromosomal Cleavage and Mutagenesis in Drosophila Using Zinc-Finger Nucleases. Genetics 161, 1169-1175 (2002).

34. Gasiunas, G., Barrangou, R., Horvath, P. \& Siksnys, V. Cas9-crRNA ribonucleoprotein complex mediates specific DNA cleavage for adaptive immunity in bacteria. Proc. Natl. Acad. Sci. U. S. A. 109, 2579-2586 (2012).

35. Jinek, M. et al. RNA-programmed genome editing in human cells. Elife 2013, 1-9 (2013). 
36. Cong, L. et al. Multiplex Genome Engineering Using CRISPR/Cas Systems. Science (80-. ). 339, 819-823 (2013).

37. Morgens, D. W., Deans, R. M., Li, A. \& Bassik, M. C. Systematic comparison of CRISPR/Cas9 and RNAi screens for essential genes. Nat. Biotechnol. 34, 634-636 (2016).

38. Grimm, D. et al. Fatality in mice due to oversaturation of cellular microRNA/short hairpin RNA pathways. Nature 441, 537-541 (2006).

39. Sledz, C. A., Holko, M., De Veer, M. J., Silverman, R. H. \& Williams, B. R. G. Activation of the interferon system by short-interfering RNAs. Nat. Cell Biol. 5, 834-839 (2003).

40. Bridge, A. J., Pebernard, S., Ducraux, A., Nicoulaz, A. L. \& Iggo, R. Induction of an interferon response by RNAi vectors in mammalian cells. Nat. Genet. 34, 263-264 (2003).

41. Fish, R. J. \& Kruithof, E. K. O. Short-term cytotoxic effects and long-term instability of RNAi delivered using lentiviral vectors. BMC Mol. Biol. 5, 1-15 (2004).

42. Hornung, V. et al. Sequence-specific potent induction of IFN-a by short interfering RNA in plasmacytoid dendritic cells through TLR7. Nat. Med.11, 263-270 (2005).

43. Judge, A. D. et al. Sequence-dependent stimulation of the mammalian innate immune response by synthetic siRNA. Nat. Biotechnol. 23, 457-462 (2005).

44. Reynolds, A. et al. Induction of the interferon response by siRNA is cell typeand duplex length-dependent. Rna 12, 988-993 (2006).

45. Jackson, A. L. et al. Expression profiling reveals off-target gene regulation by RNAi. Nat. Biotechnol. 21, 635-637 (2003).

46. Jackson, A. L. et al. Widespread siRNA 'off-target' transcript silencing mediated by seed region sequence complementarity. Rna 12, 1179-1187 (2006).

47. Lin, X. et al. siRNA-mediated off-target gene silencing triggered by a $7 \mathrm{nt}$ complementation. Nucleic Acids Res. 33, 4527-4535 (2005).

48. Birmingham, A. et al. 3' UTR seed matches, but not overall identity, are associated with RNAi off-targets. Nat. Methods 3, 199-204 (2006). 
49. Choi, S. H. et al. DUX4 recruits p300/CBP through its C-terminus and induces global H3K27 acetylation changes. Nucleic Acids Res. 44, 5161-5173 (2016).

50. Vuoristo, S. et al. DUX4 regulates oocyte to embryo transition in human. Biorxiv (2019) doi:http://dx.doi.org/10.1101/732289.

51. Jones, T. I., Himeda, C. L., Perez, D. P. \& Jones, P. L. Large family cohorts of lymphoblastoid cells provide a new cellular model for investigating

52. Banerji, C. R. S., Zammit, P. S. \& Panamarova, M. Lymphocytes contribute to DUX4 target genes in 1 FSHD muscle biopsies. Biorxiv (2019) doi:http://dx.doi.org/10.1101/717652.

54. Mulherkar, N. et al. Ebola Virus entry requires the cholesterol transporter

53. Andersson, B. S. et al. Ph-positive chronic myeloid leukemia with near-haploid conversion in vivo and establishment of a continuously growing cell line with

56. Papatheodoroua, P. et al. Lipolysis-stimulated lipoprotein receptor (LSR) is the host receptor for the binary toxin Clostridium difficile transferase (CDT). Proc. Natl. Acad. Sci. U. S. A. 108, 16422-16427 (2011).

57. Jae, L. T. et al. Deciphering the Glycosylome of Dystroglycanopathies Using Haploid Screens for Lassa Virus Entry. 340, 479-483 (2014).

58. Jae, L. T. et al. Lassa virus entry requires a trigger-induced receptor switch. Science (80-. ). 344, 1506-1510 (2014).

59. Blomen, V. A. et al. Gene essentiality and synthetic lethality in haploid human cells. Science (80-. ). 350, 1092-6 (2015).

60. Rong, Y. et al. Genome-wide screening of genes required for glycosylphosphatidylinositol biosynthesis. PLoS One 10, 1-18 (2015).

61. Wang, T. et al. Identification and characterization of essential genes in the human genome. Science (80-. ). 350, 1096-1101 (2015). 
833

834

835

836

837

838

839

840

841

842

843

844

845

846

847

848

849

850

851

852

853

854

855

856

857

858

859

860

861

862

863

864

62. Gerhards, N. M. et al. Haploid genetic screens identify genetic vulnerabilities to microtubule-targeting agents. Mol. Oncol. 12, 953-971 (2018).

63. Luteijn, R. D. et al. A Genome-Wide Haploid Genetic Screen Identifies Heparan Sulfate-Associated Genes and the Macropinocytosis Modulator TMED10 as Factors Supporting Vaccinia Virus Infection. J. Virol. 93, (2019).

64. Mezzadra, R. et al. SLFN11 can sensitize tumor cells towards IFN- $\gamma$-mediated T cell killing. PLoS One 14, 1-16 (2019).

65. Carette, J. E. et al. Generation of iPSCs from cultured human malignant cells. Blood 115, 4039-4042 (2010).

66. Olbrich, T. et al. A Chemical Screen Identifies Compounds Capable of Selecting for Haploidy in Mammalian Cells. Cell Rep. 28, 597-604.e4 (2019).

67. Bosnakovski, D. et al. Muscle pathology from stochastic low level DUX4 expression in an FSHD mouse model. Nat. Commun. 8, 1-9 (2017).

68. Agha-Mohammadi, S. et al. Second-generation tetracycline-regulatable promoter: Repositioned tet operator elements optimize transactivator synergy while shorter minimal promoter offers tight basal leakiness. J. Gene Med. 6 , 817-828 (2004).

69. Urlinger, S. et al. Exploring the sequence space for tetracycline-dependent transcriptional activators: Novel mutations yield expanded range and sensitivity. Proc. Natl. Acad. Sci. U. S. A. 97, 7963-7968 (2000).

70. Lemmers, R. J. et al. Deep characterization of a common D4Z4 variant identifies biallelic DUX4 expression as a modifier for disease penetrance in FSHD2. Eur. J. Hum. Genet. 26, 94-106 (2018).

71. De Vree, P. J. P. et al. Targeted sequencing by proximity ligation for comprehensive variant detection and local haplotyping. Nat. Biotechnol. 32, 1019-1025 (2014).

72. Chen, E. Y. et al. Enrichr: Interactive and collaborative HTML5 gene list enrichment analysis tool. BMC Bioinformatics 14, (2013).

73. Kuleshov, M. V. et al. Enrichr: a comprehensive gene set enrichment analysis web server 2016 update. Nucleic Acids Res. 44, W90-W97 (2016).

74. Bosnakovski, D. et al. The DUX4 homeodomains mediate inhibition of myogenesis and are functionally exchangeable with the Pax7 homeodomain. 
J. Cell Sci. 130, 3685-3697 (2017).

75. Banerji, C. R. S. et al. PAX7 target genes are globally repressed in facioscapulohumeral muscular dystrophy skeletal muscle. Nat. Commun. 8, (2017).

76. Robert, C. \& Watson, M. Errors in RNA-Seq quantification affect genes of relevance to human disease. Genome Biol. 16, 1-16 (2015).

77. Soutourina, J. Transcription regulation by the Mediator complex. Nat. Rev. Mol. Cell Biol. 19, 262-274 (2018).

78. Shadle, S. C. et al. DUX4-induced dsRNA and MYC mRNA stabilization activate apoptotic pathways in human cell models of facioscapulohumeral dystrophy. PLoS Genet. 13, 1-25 (2017).

79. Tawil, R. et al. Evidence-based guideline summary: Evaluation, diagnosis, and

80. Essletzbichler, P. et al. Megabase-scale deletion using CRISPR/Cas9 to generate a fully haploid human cell line. Genome Res. 24, 2059-2065 (2014).

81. Lek, A. et al. Applying genome-wide CRISPR-Cas9 screens for therapeutic discovery in facioscapulohumeral muscular dystrophy. Sci. Transl. Med. 12, 911 (2020).

82. Banerji, C. R. S. et al. $\beta$-catenin is central to DUX4-driven network rewiring in facioscapulohumeral muscular dystrophy. J. R. Soc. Interface 12, (2015).

83. Tsumagari, K. et al. Gene expression during normal and FSHD myogenesis. BMC Med. Genomics 4, (2011).

84. Cullot, G. et al. CRISPR-Cas9 genome editing induces megabase-scale chromosomal truncations. Nat. Commun. 10, 1-14 (2019).

85. Dull, T. et al. A third-generation lentivirus vector with a conditional packaging system. J. Virol. 72, 8463-71 (1998).

86. Shen, M. W. et al. Predictable and precise template-free CRISPR editing of pathogenic variants. Nature 563, 646-651 (2018).

894 87. Urasaki, A., Morvan, G. \& Kawakami, K. Functional dissection of the Tol2 transposable element identified the minimal cis-sequence and a highly repetitive sequence in the subterminal region essential for transposition. 
Genetics 174, 639-649 (2006).

88. Ferreboeuf, M. et al. DUX4 and DUX4 downstream target genes are expressed in fetal FSHD muscles. Hum. Mol. Genet. 23, 171-181 (2014).

89. Borgström, A. et al. A novel multiplex qPCR targeting 23S rDNA for diagnosis of swine dysentery and porcine intestinal spirochaetosis. BMC Vet. Res. 13, 18 (2017).

90. van Attekum, M., Terpstra, S., Reinen, E., Kater, A. \& Eldering, E. Macrophage-mediated chronic lymphocytic leukemia cell survival is independent of APRIL signaling. Cell Death Discov. 2, (2016).

91. Hashimshony, T., Wagner, F., Sher, N. \& Yanai, I. CEL-Seq: Single-Cell RNASeq by Multiplexed Linear Amplification. Cell Rep. 2, 666-673 (2012).

92. Grün, D. et al. Single-cell messenger RNA sequencing reveals rare intestinal cell types. Nature 525, 251-255 (2015).

93. Hashimshony, T. et al. CEL-Seq2: Sensitive highly-multiplexed single-cell RNA-Seq. Genome Biol. 17, 1-7 (2016).

94. Love, M. I., Huber, W. \& Anders, S. Moderated estimation of fold change and dispersion for RNA-seq data with DESeq2. Genome Biol. 15, 1-21 (2014).

95. Sanson, K. R. et al. Optimized libraries for CRISPR-Cas9 genetic screens with multiple modalities. Nat. Commun. 9, 1-15 (2018).

96. Wolfe, D., Dudek, S., Ritchie, M. D. \& Pendergrass, S. A. Visualizing genomic information across chromosomes with PhenoGram. BioData Min. 6, 1-12 (2013). 


\section{Supplementary figures}

921

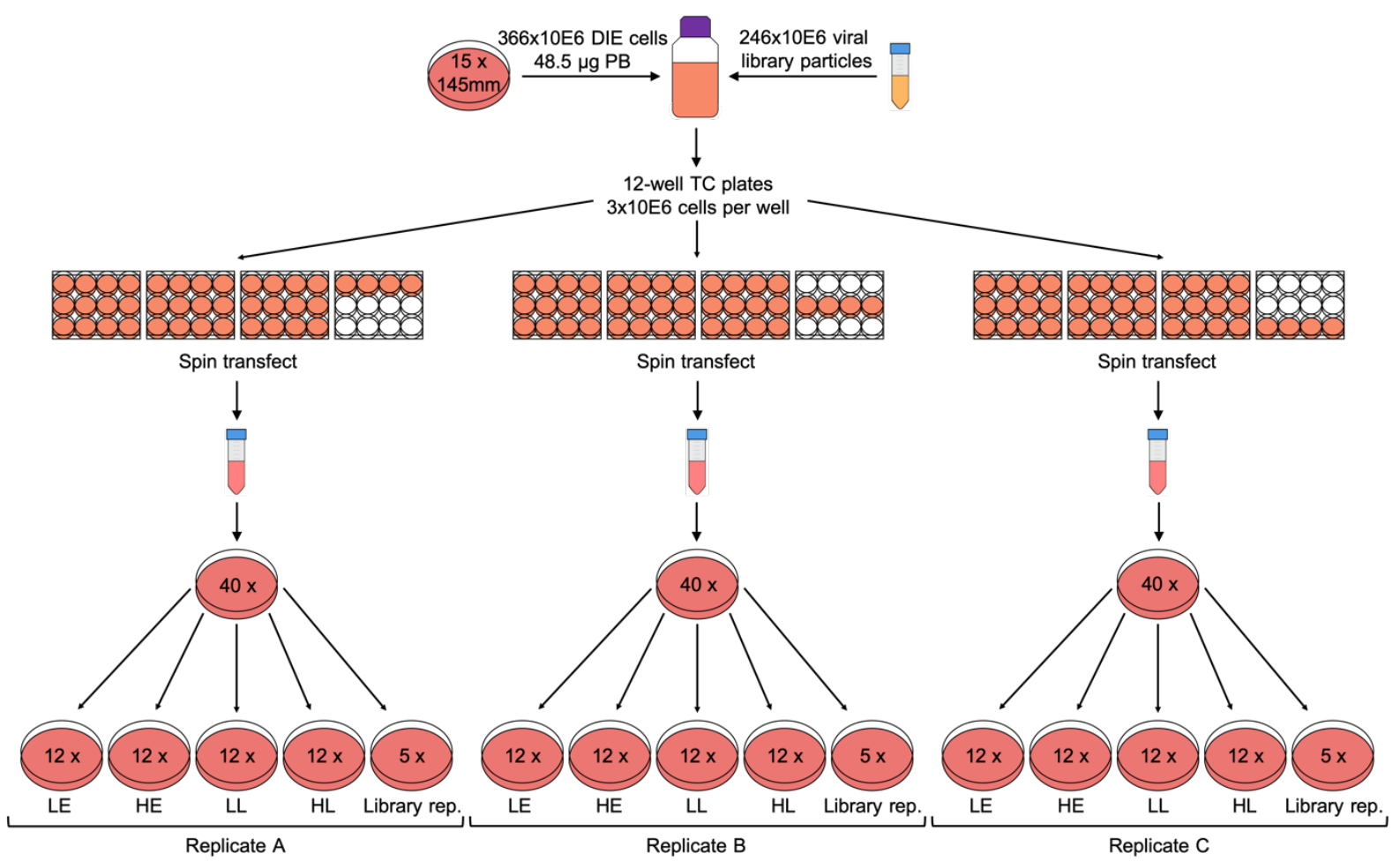

922 Supplementary figure S1. Execution of the CRIPSR/Cas9 genome wide screens. A schematic

923 representation of the execution of the CRISPR/Cas9 screens. PB: Polybrene, TC: Tissue culture, LE:

924 Low doxycycline/Early harvest, HE: High doxycycline/Early harvest, LL: Low doxycycline/Late harvest,

925 HL: High doxycycline/Late harvest, Library rep: Library representation.

\section{Phase contrast}
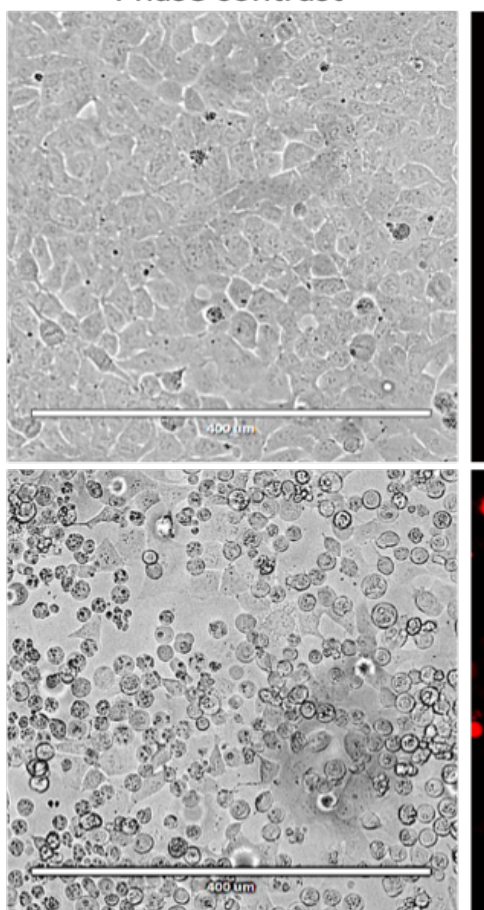

PI
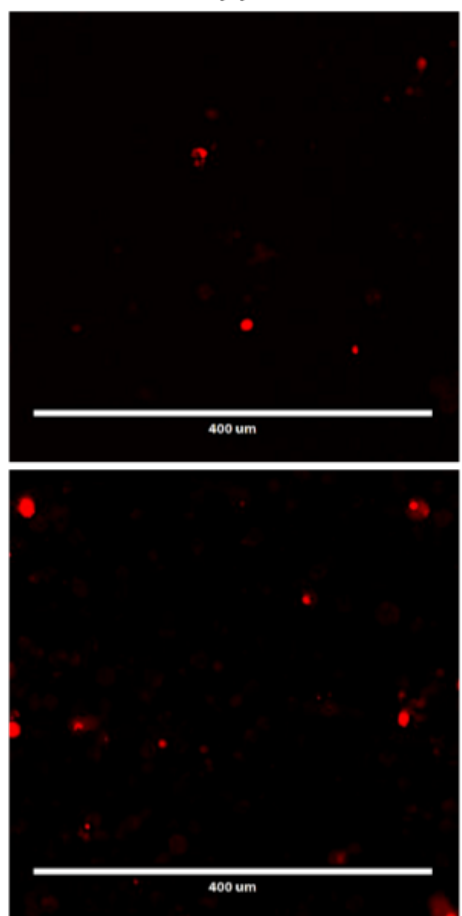

Annexin V

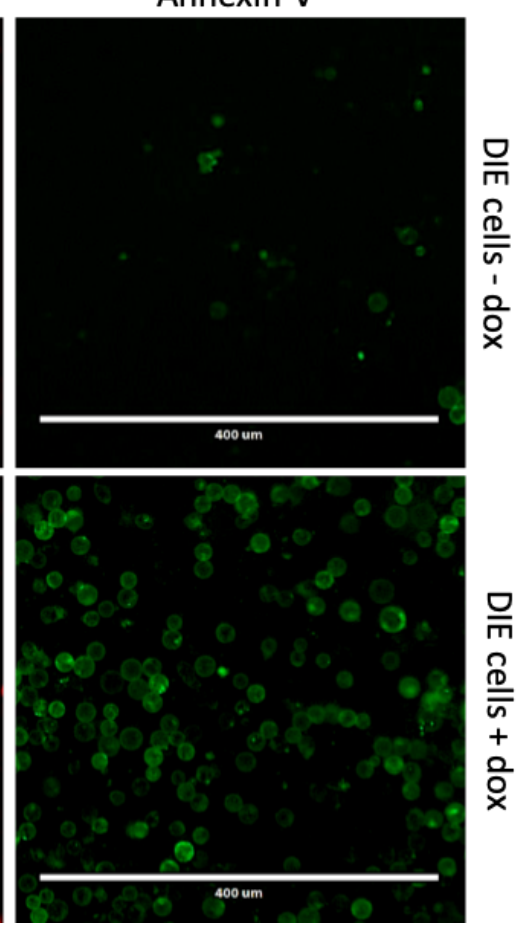


bioRxiv preprint doi: https://doi.org/10.1101/2020.07.27.223420; this version posted July 27, 2020. The copyright holder for this preprint

(which was not certified by peer review) is the author/funder, who has granted bioRxiv a license to display the preprint in perpetuity. It is made available under aCC-BY-NC-ND 4.0 International license.

928 Supplementary figure S2. Living and dying DIE cells. Uninduced (top panel) and doxycycline

929 induced (bottom panel) DIE cells, stained with Propidium lodide (PI) (middle panel) and AnnexinV-Alexa

930 Fluor 488 (right panel), with a phase contrast image in the left panel. DIE cells in the bottom panel are

931 stained positive for AnnexinV, with no increasing PI signal compared to uninduced DIE cells (top panel).

932

933

Early low

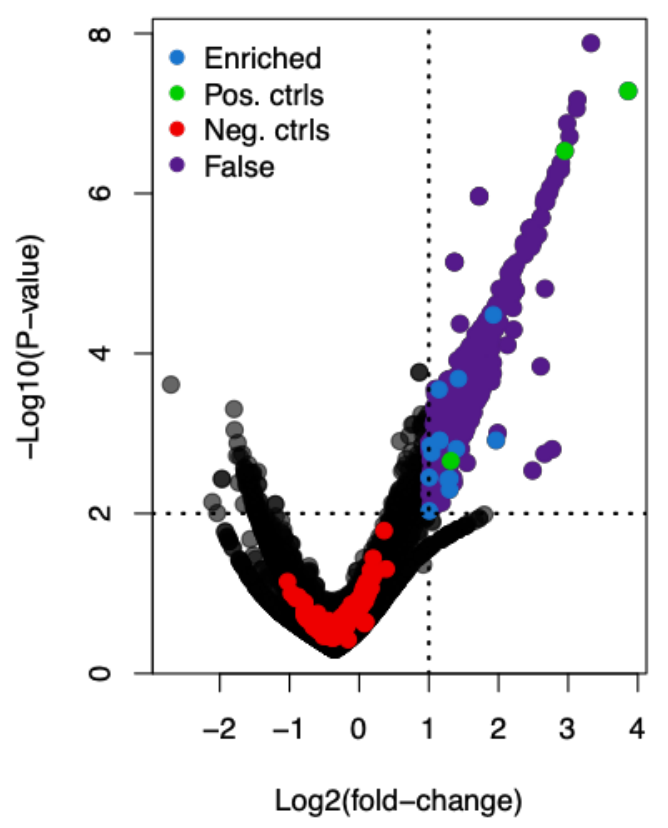

Late low

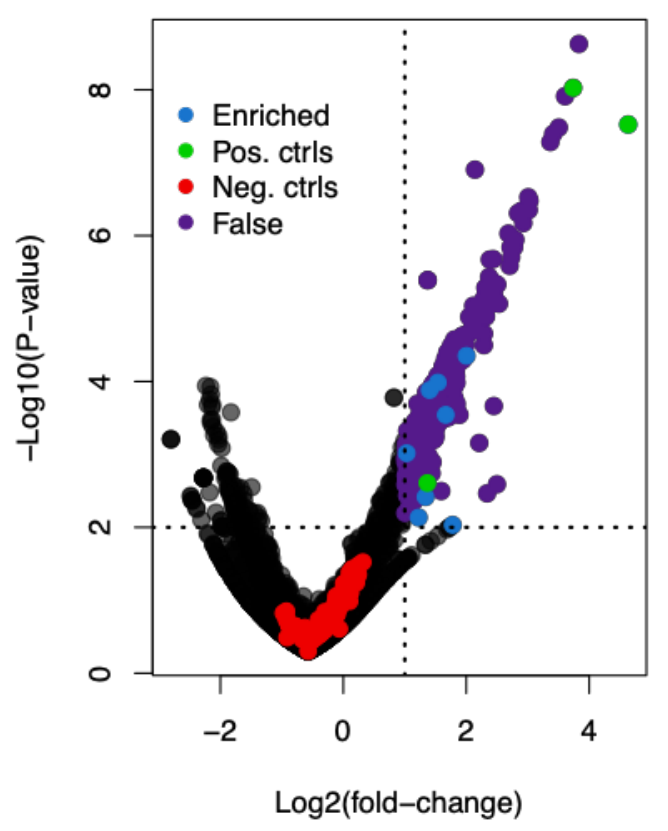

\section{Early high}

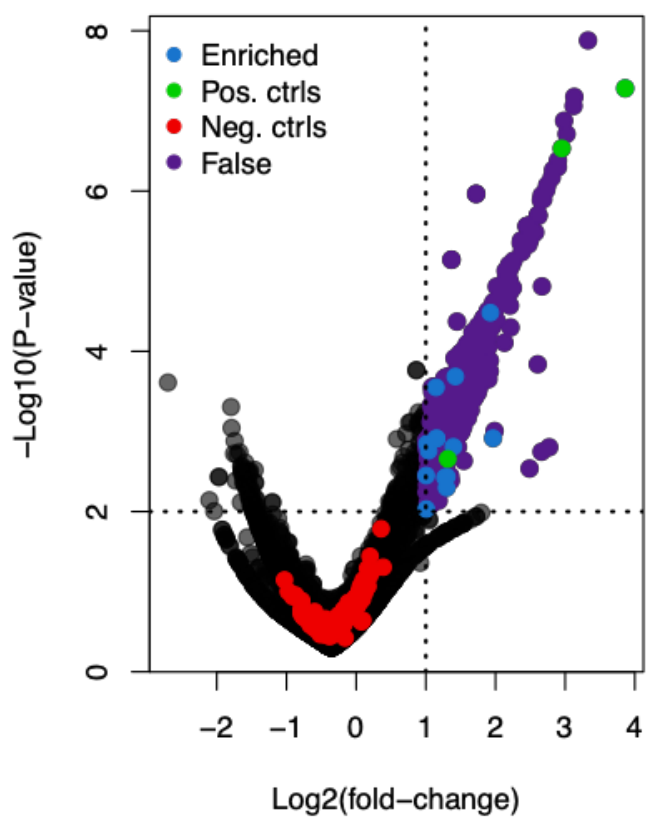

Late high

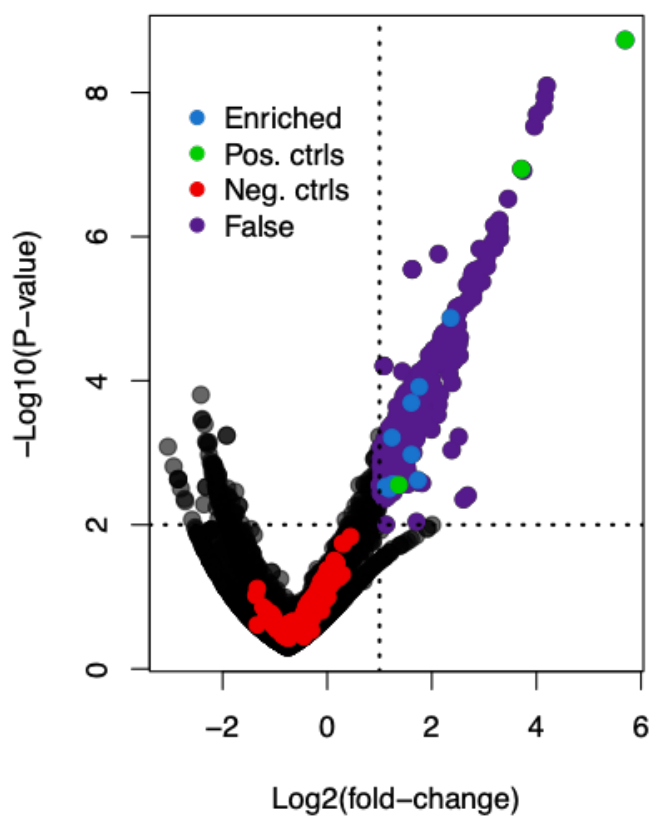


935 Supplementary figure S3. Analysis of enriched gRNAs from screen data processed with a one-

936 sided analysis. Volcano plots illustrating enrichment of gRNAs in the surviving population of DIE cells

937 of all 4 screens. Due to the one-sided analysis, depletion data should not be taken into consideration.

938 For a two-sided analysis see supplementary figure S6. The Log2(foldchange) (log2FC) is plotted on the

$939 \mathrm{X}$-axis and the -Log10(p-value), (-log10PV) is plotted on the Y-axis. Data shown here shows the

940 average log2FC and -log10PV of each guide set (set: 4 guides per gene). Blue points represent guide

941 sets that are significantly enriched in this data set $(\log 2 F C \geq 1,-\log 10 P V \geq 2)$, purple points represent

942 the false positive hits that on chromosome $5 q$ and chromosome $19 p$, green point are the positive

943 controls (DUX4, MAST1, MGAT4B), red points represent the Non-Target control guides.

945 Supplementary figure S4. PhenoGrams showing enriched hits in the human genome.

946 Chromosomal ideogram indicating the location of enriched hits in the human genome, for each of the 4

947 screens. PhenoGram is a software created by the Ritchie lab from the university of Pennsylvania96. 
bioRxiv preprint doi: https://doi.org/10.1101/2020.07.27.223420; this version posted July 27, 2020. The copyright holder for this preprint

(which was not certified by peer review) is the author/funder, who has granted bioRxiv a license to display the preprint in perpetuity. It is made available under aCC-BY-NC-ND 4.0 International license.

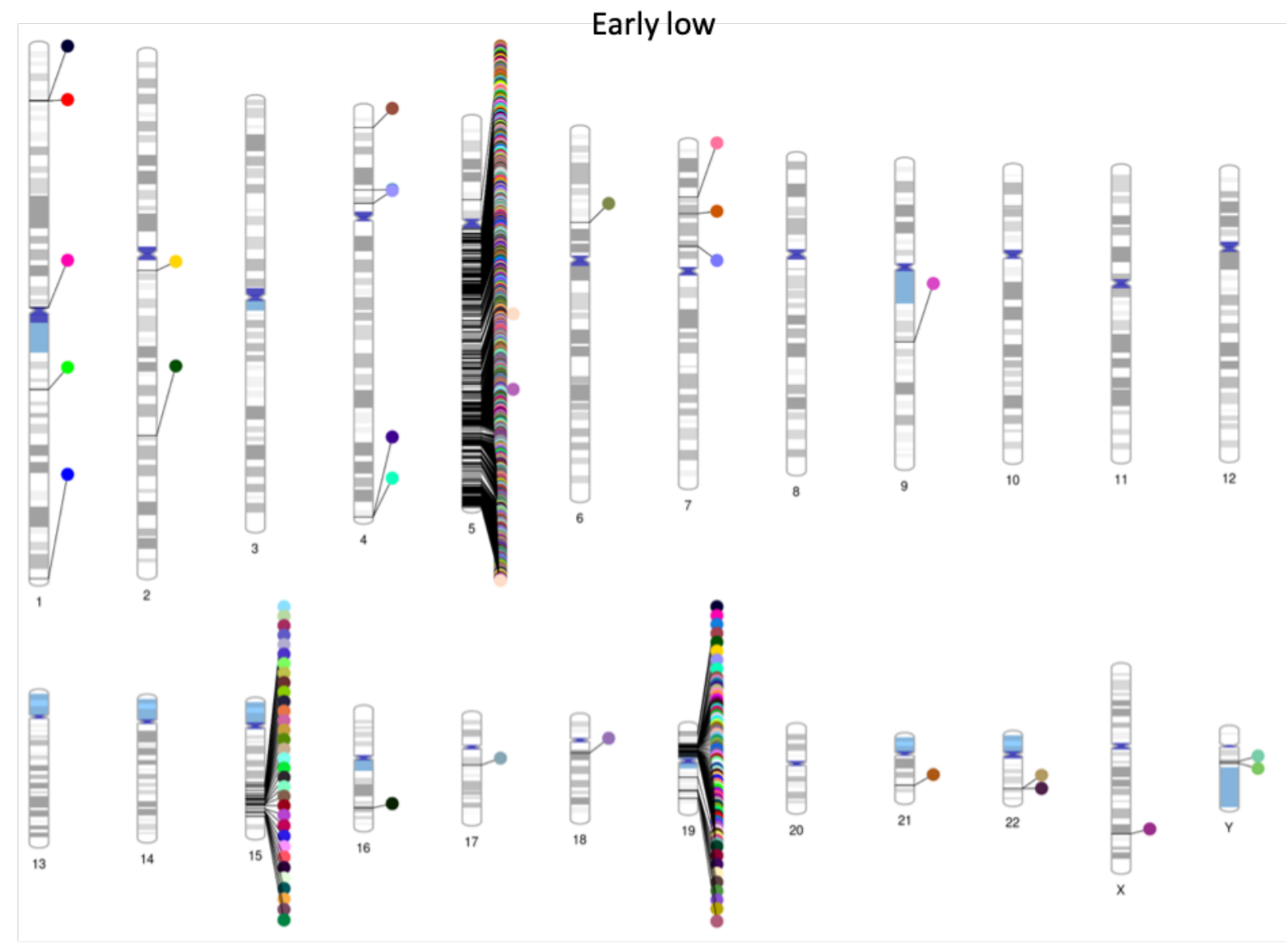

\section{Early high}
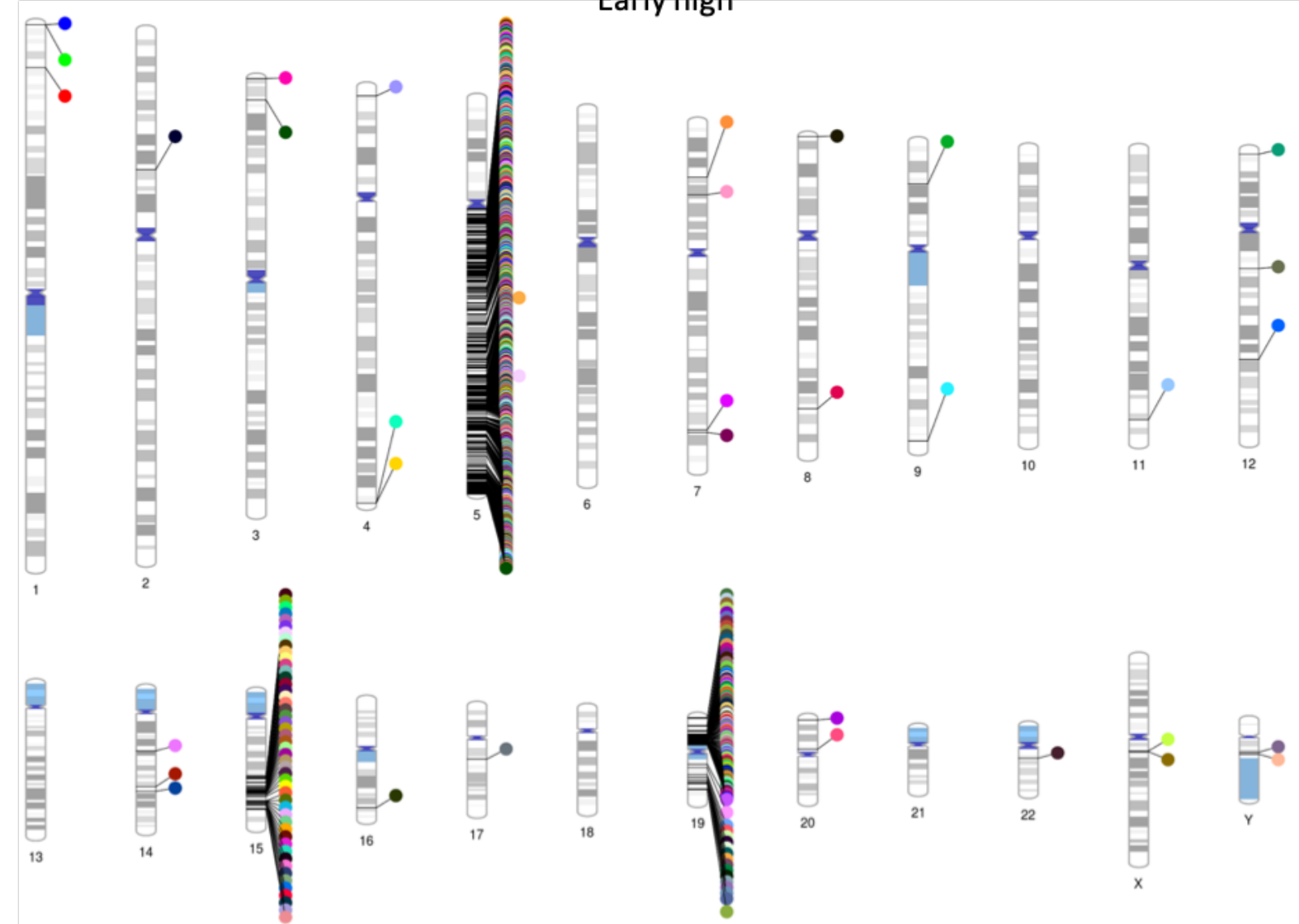
bioRxiv preprint doi: https://doi.org/10.1101/2020.07.27.223420; this version posted July 27, 2020. The copyright holder for this preprint

(which was not certified by peer review) is the author/funder, who has granted bioRxiv a license to display the preprint in perpetuity. It is made available under aCC-BY-NC-ND 4.0 International license.
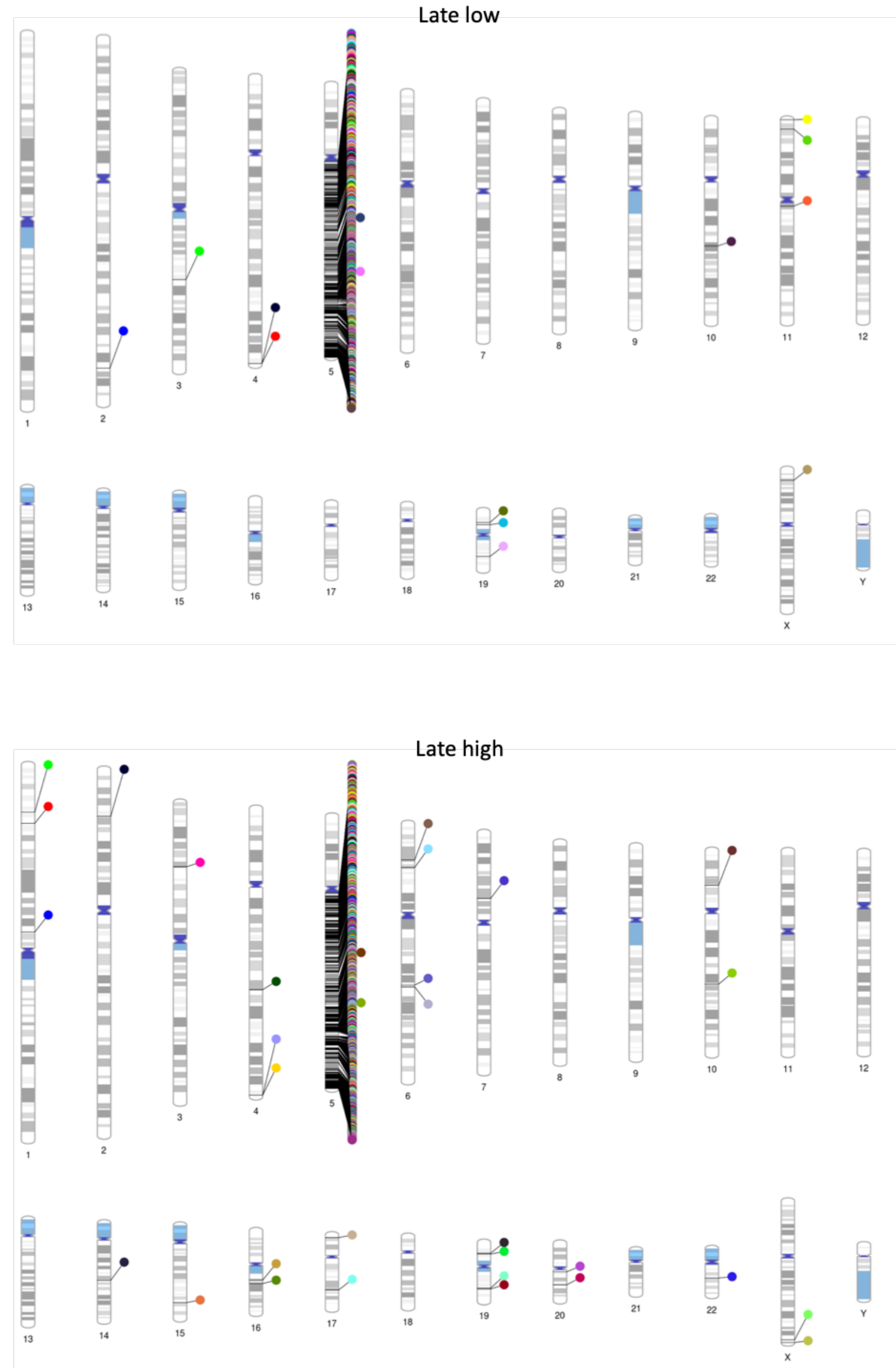
951

952

953

960

961

962

963

970

971

972

973

974

975

976

977
High doxycycline
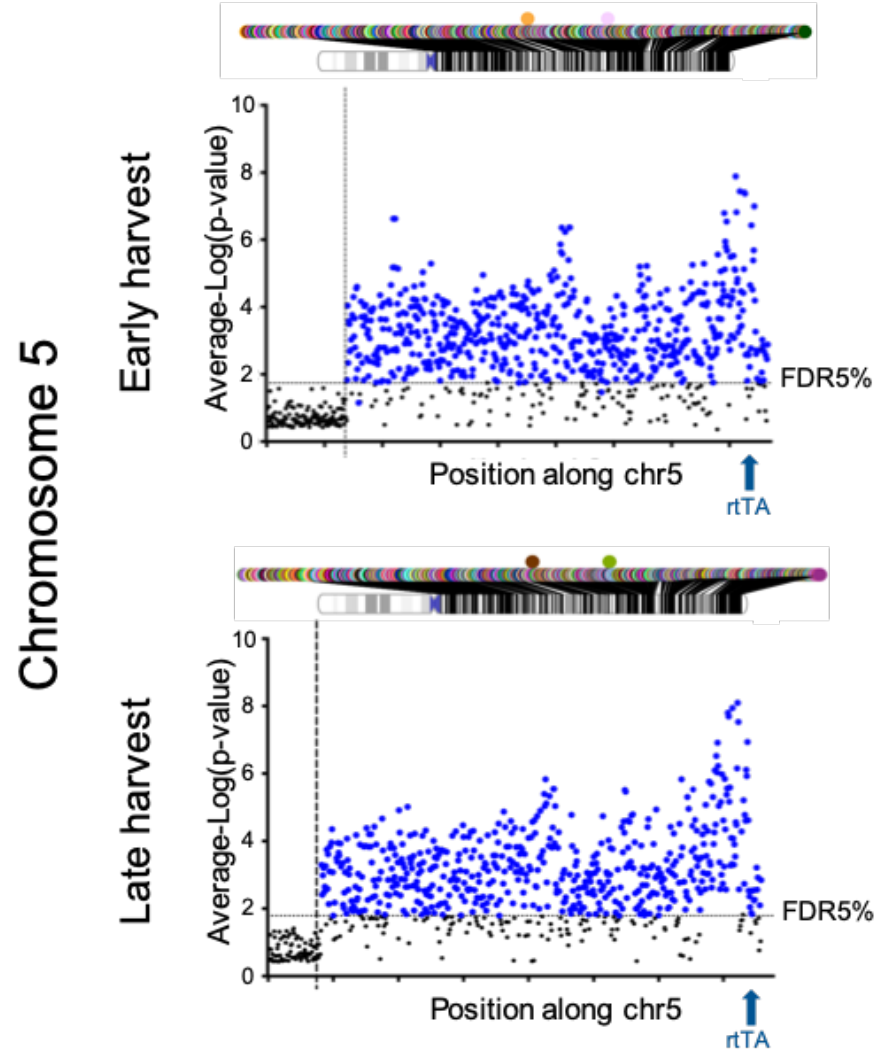

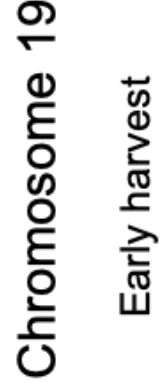

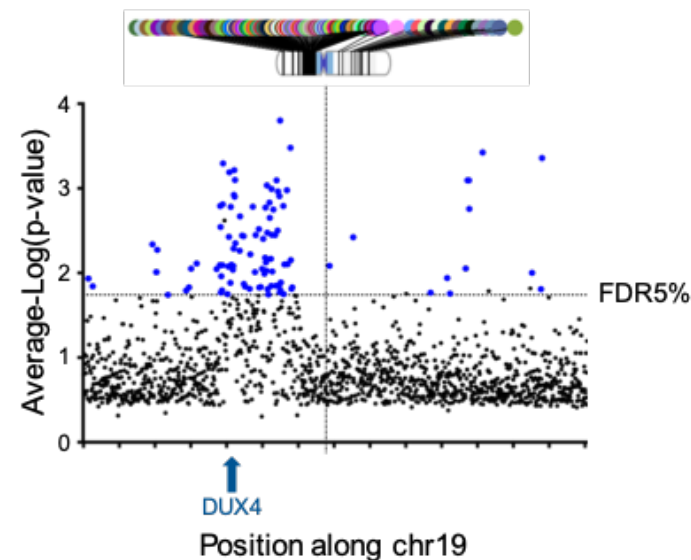

Position along chr19

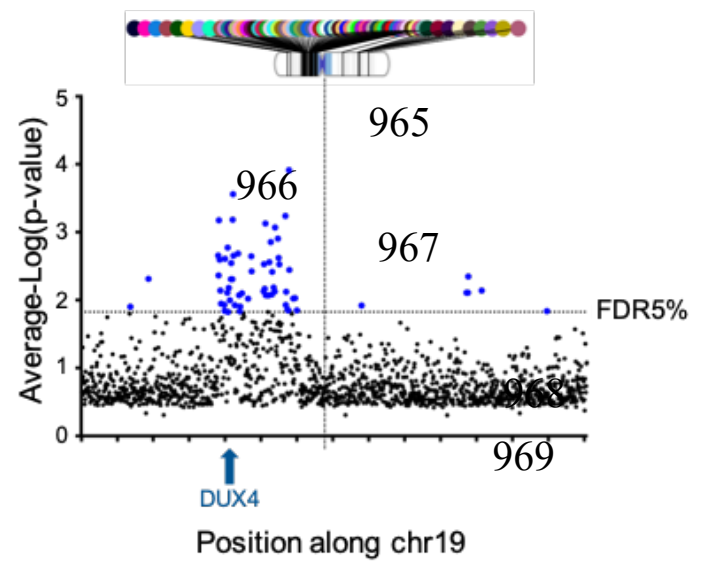

Low doxycycline
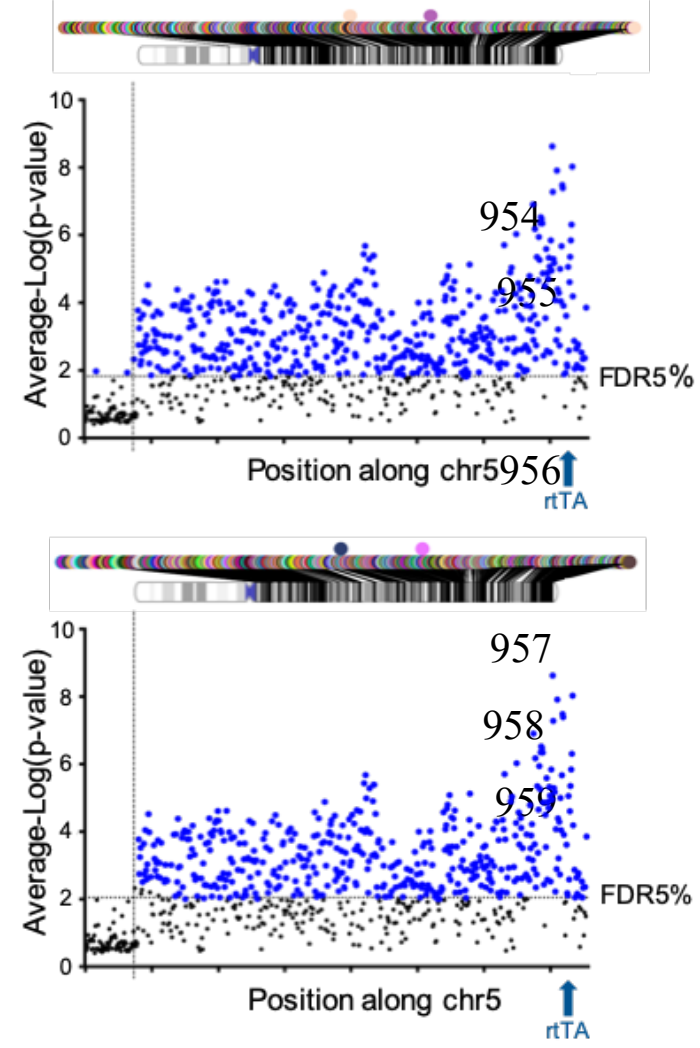

Position along chr19

\section{Supplementary figure S5. Data plot displaying enriched hits on chromosome $5 q$ and} chromosome 19. The average-Log(p-value) is plotted on the $\mathrm{Y}$-axis, and the $\mathrm{X}$-axis is displaying the position on the chromosome. The vertical abline indicates the position of the centromere. All points above the horizontal abline (in blue) indicating significantly enriched hits that fall below the $5 \%$ False Discovery Rate (FDR) threshold. The location of the transgene is annotated with a blue arrow on the 

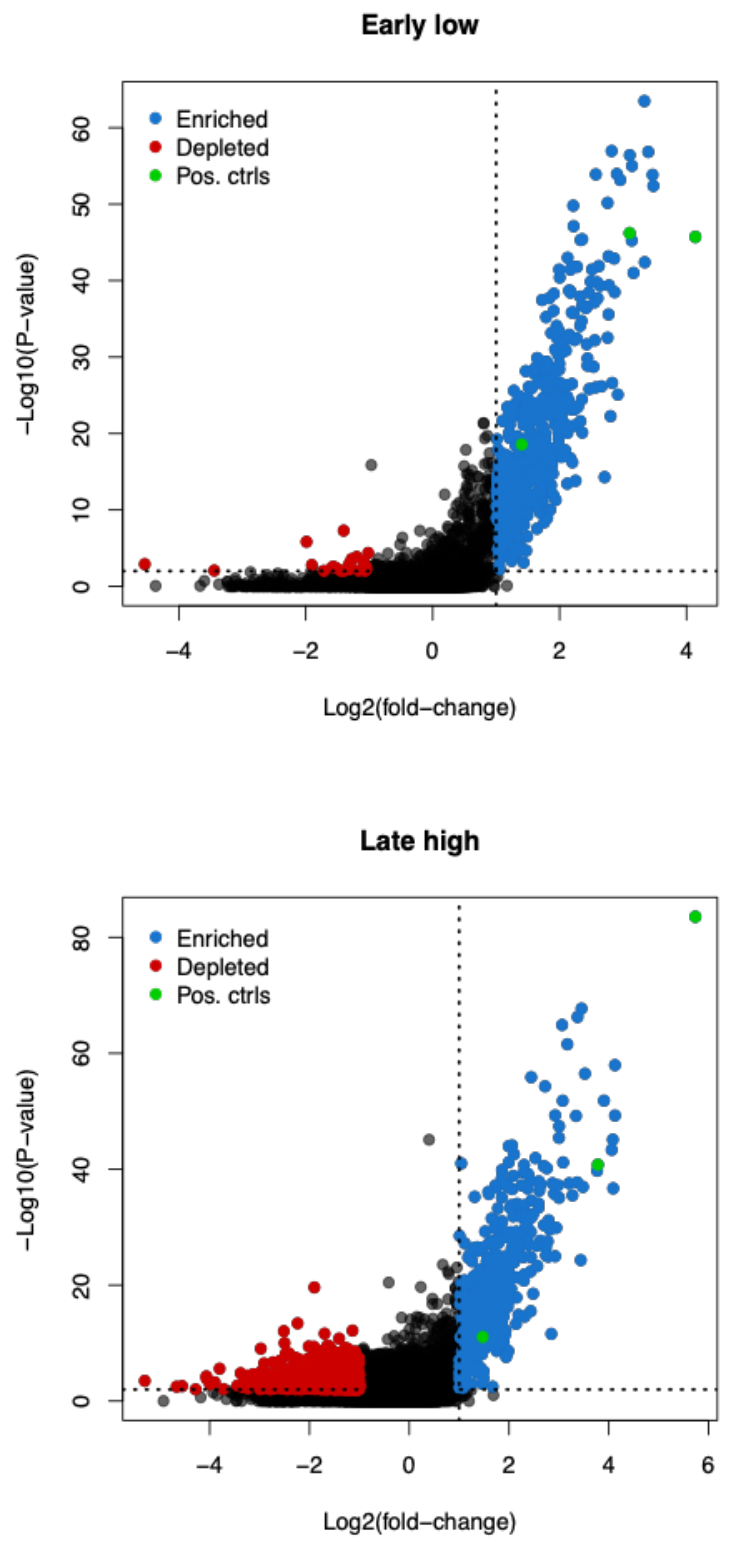
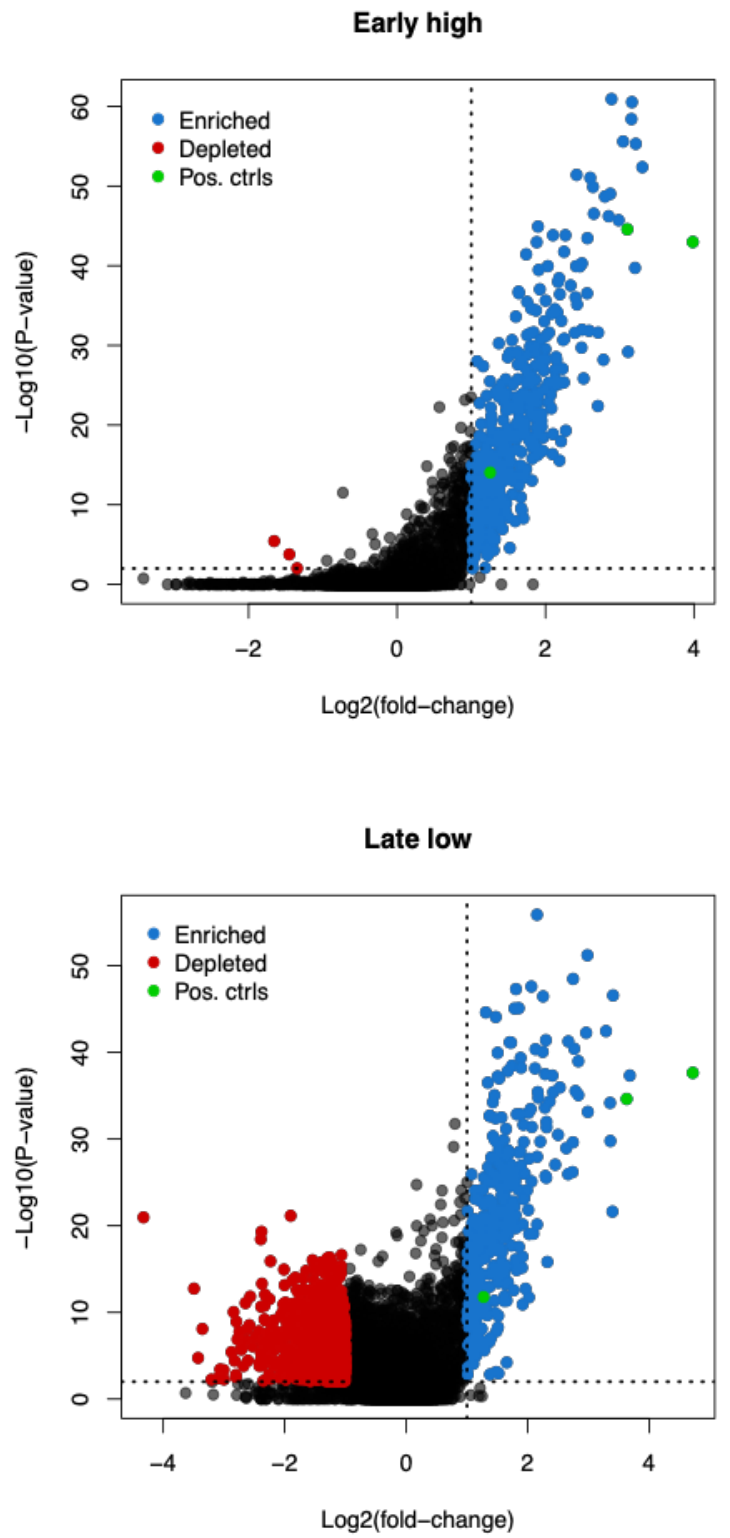

979 Supplementary figure S6. Analysis of enriched and depleted gRNAs from screen data analyzed

980 in a two-sided analysis. Volcano plots illustrating enrichment and depletion of gRNAs in all 4 screens.

981 The Log2(foldchange) (log2FC) is plotted on the X-axis and the -Log10(p-value), (-log10PV) is plotted 982 on the Y-axis. Dots indicated in blue are significantly enriched gRNAs sequences (Log2FC $\geq 1$, $983 \log 10 \mathrm{PV} \geq 2$ ). Green dots represent positive controls (DUX4, MAST1, MGAT4B), and red dots represent 984 the depleted targets. 

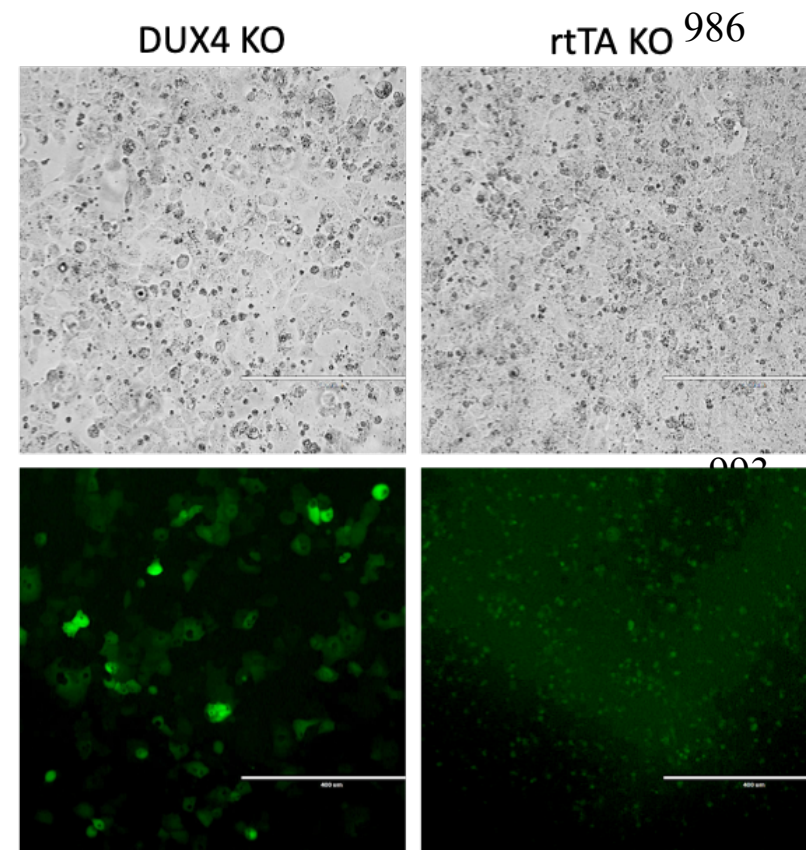

Supplementary figure S7. Individual knock-outs in DIE-ieGFP-Cas9 cells demonstrating eGFP activation in cells with a functional TetO inducible system. Phase contrast (top panel) and fluorescent images (bottom panel) of DIE cells containing a DUX4 KO (left panel), and rtTA KO (right panel) induced with $250 \mathrm{ng} / \mathrm{ml}$ doxycycline. DIE cells had reached over $100 \%$ confluency.

999

A
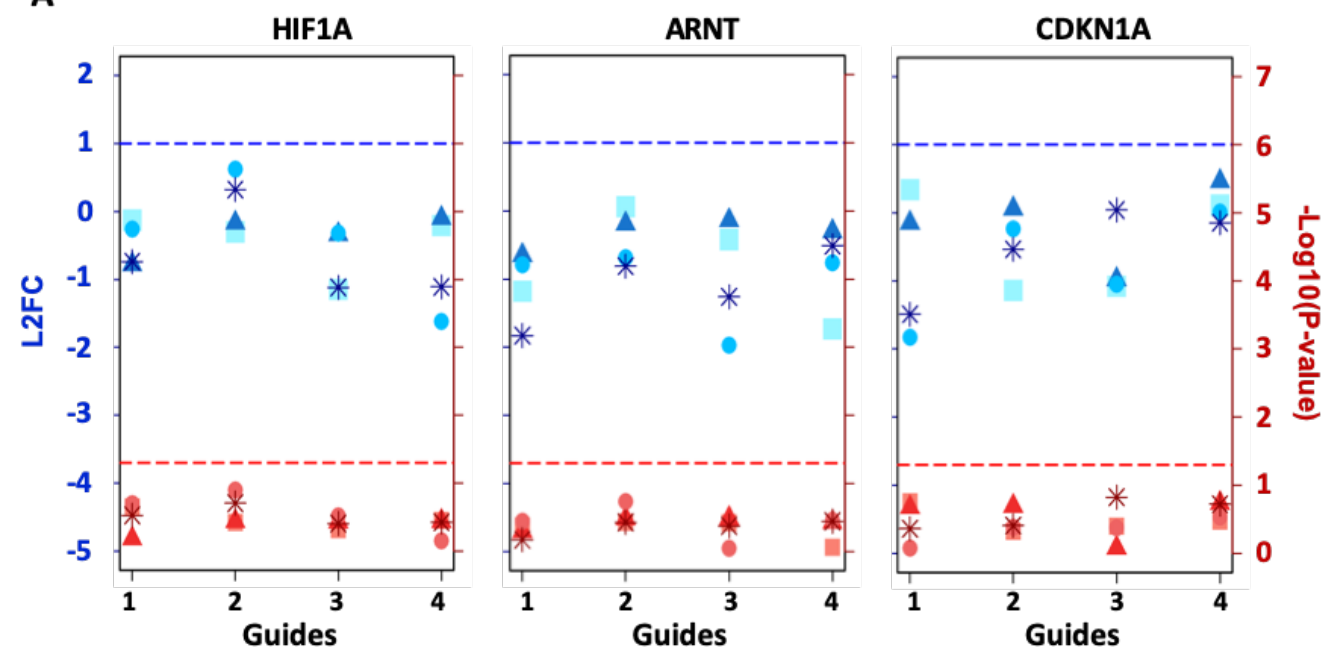

- Early low

$\Delta$ Early high

- Late low

* Late high

B

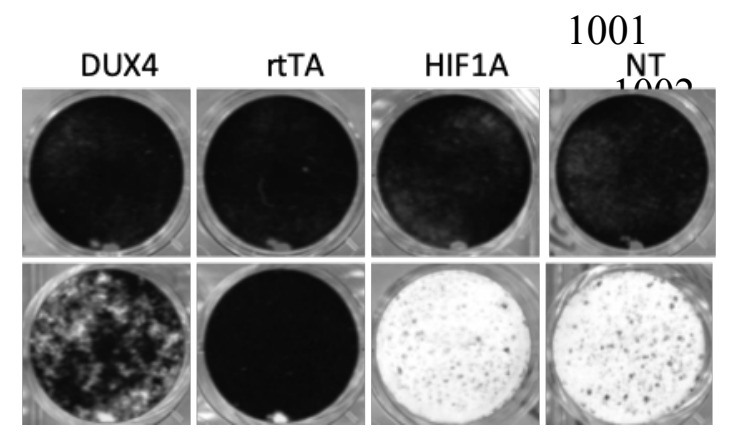

Supplementary figure S8. Validation of genes involved in the HIF1 hypoxia pathway (A) Data plots showing the enrichment of sgRNA targeting HIF1A. HIF1B/ARNT, CDKN1A. The LFC value of each individual guide is plotted on the left $y$-axis, indicated in blue, and the -Log10 $\mathrm{P}$-value is plotted on the right $\mathrm{y}$-axis, in red.

1008 Guides located above the blue and red intermitted ablines are considered significant (blue: LFC > 1, 1009 red: -Log10 P-value > 1.3). (B) Viability staining of DIE-Cas9 uninduced (top panel) and induced with $1010100 \mathrm{ng} / \mathrm{ml}$ doxycycline (lower panel) cells, transfected with DUX4, rtTA, HIF1A and non-targeting (NT) 1011 sgRNA coding plasmids. 